TRANSACTIONS OF THE

AMERICAN MATHEMATICAL SOCIETY

Volume 353, Number 9, Pages 3741-3767

S 0002-9947(01)02710-6

Article electronically published on May 4, 2001

\title{
CANONICAL SYMBOLIC DYNAMICS FOR ONE-DIMENSIONAL GENERALIZED SOLENOIDS
}

\author{
INHYEOP YI
}

\begin{abstract}
We define canonical subshift of finite type covers for Williams' onedimensional generalized solenoids, and use resulting invariants to distinguish some closely related solenoids.
\end{abstract}

\section{Introduction}

R. F. Williams has developed a theory of expanding attractors for a dynamical system ([14, 15, 16]). These can be modeled as shift maps of generalized $n$-solenoids which are defined as inverse limits of immersions of $n$-dimensional branched manifolds satisfying certain axioms.

In this paper, we produce canonical shift of finite type (SFT) covers of Williams' 1-solenoid systems in the following sense: Let $\bar{X}$ be a 1-solenoid, $\bar{f}$ a shift map on $\bar{X}$, and $\overline{\mathcal{O}}$ a union of finitely many periodic orbits of $\bar{f}$. We give an algorithm which takes the input $\{\bar{X}, \bar{f}, \overline{\mathcal{O}}\}$ and produces as output a mixing SFT $\Sigma_{\mathcal{O}}$ with shift map $\sigma_{\mathcal{O}}$ and a semiconjugacy $p_{\mathcal{O}}: \Sigma_{\mathcal{O}} \rightarrow \bar{X}$ (that is, $p_{\mathcal{O}}$ is a continuous surjection and $\left.p_{\mathcal{O}} \circ \sigma_{\mathcal{O}}=\bar{f} \circ p_{\mathcal{O}}\right)$. Then we prove that if there is a conjugacy $\phi$ of 1-solenoid systems $(\bar{X}, \bar{f})$ and $(\bar{Y}, \bar{g})$ such that $\phi$ sends $\overline{\mathcal{O}}$ to $\overline{\mathcal{O}}^{\prime}$, then there is a unique conjugacy $\tilde{\phi}$ such that $\phi \circ p_{\mathcal{O}}=p_{\mathcal{O}^{\prime}} \circ \tilde{\phi}$. These covers can be exploited to give nontrivial computable invariants distinguishing closely related solenoid systems.

To our knowledge, we are giving the first construction of canonical SFT covers for a class of nonzero-dimensional systems. The canonicalness requires the dependence on $\mathcal{O}$, and perhaps this is why it was not noticed earlier. However, there have been other works (11, 11) achieving some specialness or invariant for SFT covers of some systems, and there were earlier constructions of canonical covers for some systems. Krieger ([ $]$ ) gave canonical SFT covers of sofic systems (which are zero-dimensional), and Fried (7]) more generally offered canonical coordinate (CC) covers of finitely presented (FP) systems. These covers are built from sets of possible pasts and futures. The Krieger-Fried covers make the step from FP to CC. The 1-solenoid systems are already CC, and the covers we produce are SFTs. We raise the question, can our relatively simple one-dimensional construction be generalized in some inductive way to produce canonical symbolic dynamics for higher dimensional generalized solenoid systems?

Received by the editors September 14, 1999 and, in revised form, April 6, 2000.

2000 Mathematics Subject Classification. Primary 58F03, 58F12; Secondary 05C20, 54F50, $58 \mathrm{~F} 15$.

Key words and phrases. Generalized solenoid, shift of finite type, shift equivalence, canonical SFT cover, elementary presentation, Bowen-Franks group. 
Apart from the matter of canonical symbolic dynamics, we mention renewed interest in Williams' systems and related systems on account of connections with ordered group invariants $([3,12,18,19])$ and substitutions and tilings ([2, 6]).

We study the 1-solenoid systems as purely topological systems. For this we give some defining topological axioms closely related to Williams' axioms. A 1solenoid of Williams becomes one of our 1-solenoids by ignoring the differentiable structure. Conversely, every topologically defined 1-solenoid can be given a differentiable structure which makes it a 1-solenoid in the sense of Williams. However, the essential aspects of the situation are not differentiable but topological, and to clarify this we give the purely topological development.

The outline of the paper is as follows. In Section 2, following Williams (14, 15) rather closely, we give axioms for our systems and prove some basic facts about them. We also recall the construction of an SFT cover from a graph presentation. In Section 3, we recall Williams' definition of shift equivalence and show that every topological conjugacy of shift maps on branched 1-solenoids is induced by a shift equivalence of their graph presentations. (A 'branched' solenoid is a solenoid derived from a presentation which need not satisfy Williams' Flattening Axiom, so this is a slight generalization of Williams' work.) We also establish a key observation: If the shift equivalence is given by graph maps (maps sending vertices to vertices), then the conjugacy lifts uniquely to a conjugacy of the SFT covers derived from the graph presentations. In Section 4 , given $\overline{\mathcal{O}}$, we give a graph algorithm for a new graphical presentation $\left(X_{\mathcal{O}}, f_{\mathcal{O}}\right)$ of the solenoid system. One consequence of this construction is that every 1-solenoid system with a fixed point has an elementary presentation in the sense of Williams, so this extends Williams' classification result $([15, \S 7])$ to all 1-solenoid systems with fixed points. In Section 5, we use the previous results to produce the canonical SFT covers, and use them to distinguish the pair of systems considered by Williams and Ustinov ([13, 15]) by computing Bowen-Franks groups of certain attached canonical SFT covers. In Appendix A, we show that our canonical SFT covers are not canonical as one-sided SFTs, despite the one-sided aspects of the construction. In Appendix B, we show that our graph presentations of topological 1-solenoid systems can be given a differentiable structure making them branched manifolds with differentiable immersions in the sense of Williams.

\section{Markov maps AND their SFT COVERS}

In the style of Williams ([14, 15]), we will define several axioms which might be satisfied by a continuous self-map of a directed graph. Let $X$ be a directed graph with vertex set $\mathcal{V}$ and edge set $\mathcal{E}$, and $f: X \rightarrow X$ a continuous map. Axioms 0-3 and 5 correspond to Williams' Axioms $0-2,3^{\circ}$, and 4 in [15].

Axiom 0. (Indecomposability) $(X, f)$ is indecomposable.

Axiom 1. (Nonwandering) All points of $X$ are nonwandering under $f$.

Axiom 2. (Flattening) There is $k \geq 1$ such that for all $x \in X$ there is an open neighborhood $U$ of $x$ such that $f^{k}(U)$ is homeomorphic to $(-\epsilon, \epsilon)$.

Axiom 3. (Expansion) There are a metric $d$ compatible with the topology and positive constants $C$ and $\lambda$ with $\lambda>1$ such that for all $n>0$ and all points $x, y$ on a common edge of $X$, if $f^{n}$ maps the interval $[x, y]$ into an edge, then $d\left(f^{n} x, f^{n} y\right) \geq C \lambda^{n} d(x, y)$.

Axiom 4. (Nonfolding) $\left.f^{n}\right|_{X-\mathcal{V}}$ is locally one-to-one for every positive integer $n$. 
Axiom 5. (Markov) $f(\mathcal{V}) \subseteq \mathcal{V}$.

Remarks 2.1. (1) Axiom 0 means that $X$ cannot be split into two nonempty, closed, $f$-invariant subsets $([15, \S 1])$.

(2) It will be convenient to think of the connection map $f: X \rightarrow X$ satisfying the Axioms 0-5 (except possibly Axiom 2) as increasing arclength by a positive constant factor. For this, we (tacitly) replace the given metric $d$ with an equivalent metric $d^{\prime}$ defined as follows: Williams' construction ([15, 6.2]) gives a (unique) measure $\mu$ for which there is a constant $\lambda>1$ such that $\mu(X)=1$ and $\mu(f(I))=\lambda \mu(I)$ for every small interval $I \subset X$. Define $d^{\prime}(x, y)$ to be the measure of the smallest interval from $x$ to $y$, and define the arclength of an embedded interval to be its measure.

Standing Assumption. In this paper, we always assume that $(X, f)$ satisfies Axioms 0 and 1 .

For a given directed graph $X$ with a continuous map $f: X \rightarrow X$, let $\bar{X}$ be the inverse limit space

$$
\bar{X}=X \stackrel{f}{\longleftarrow} X \stackrel{f}{\longleftarrow} \cdots=\left\{\left(x_{0}, x_{1}, x_{2}, \ldots\right) \in \prod_{0}^{\infty} X \mid f\left(x_{n+1}\right)=x_{n}\right\},
$$

and $\bar{f}: \bar{X} \rightarrow \bar{X}$ the induced homeomorphism defined by

$$
\left(x_{0}, x_{1}, x_{2}, \ldots\right) \mapsto\left(f\left(x_{0}\right), f\left(x_{1}\right), f\left(x_{2}\right), \ldots\right)=\left(f\left(x_{0}\right), x_{0}, x_{1}, \ldots\right) .
$$

Let $Y$ be a topological space and $g: Y \rightarrow Y$ a homeomorphism. We call $Y$ a onedimensional generalized solenoid or 1-solenoid and $g$ a shift map if there exist a directed graph $X$ and a graph map $f: X \rightarrow X$ such that $(X, f)$ satisfies all six Axioms and $(\bar{X}, \bar{f})$ is topologically conjugate to $(Y, g)$. We say that $(X, f)$ is a presentation of $(Y, g)$. If $(X, f)$ satisfies all axioms except possibly the Flattening Axiom, then we call $Y$ a branched solenoid.

Remarks 2.2. (1) Williams defined an $n$-dimensional generalized solenoid $\bar{X}$ and a shift map $\bar{f}$ as the inverse limit of a system $(X, f)$ satisfying Axioms 0-3 where $X$ is a differentiable $n$-dimensional branched manifold and $f: X \rightarrow X$ is an immersion ([14, 15, 16]). We generalize his systems in the topological category for the one-dimensional case. As a topological system, every 1solenoid of Williams is a 1-solenoid by our topological definition. We will see the relation between Williams' definition and the topological definition in Appendix B.

(2) The Nonfolding Axiom is the topological condition we use in place of the differentiable immersion condition.

(3) If $(X, f)$ satisfies Axioms $0-4$, then there is a presentation $\left(X^{\prime}, f^{\prime}\right)$ satisfying Axioms 0-5 such that $(\bar{X}, \bar{f})$ is topologically conjugate to $\left(\overline{X^{\prime}}, \overline{f^{\prime}}\right)([14$, Proposition 3.5]). Williams proved [14, 3.5] assuming the immersion condition, but his proof goes through with our Axioms 0-4.

Example 2.3. Let $X$ be the unit circle on the complex plane. Suppose that 1 and -1 are the vertices of $X$, and that the upper half circle $e_{1}$ and the lower half circle $e_{2}$ with counterclockwise direction are the edges of $X$. Define $f, g: X \rightarrow X$ by

$$
f: z \mapsto z^{2} \text { and } g: z \mapsto \begin{cases}z^{2} & \text { if } z \in e_{1} \\ z^{-2} & \text { if } z \in e_{2}\end{cases}
$$



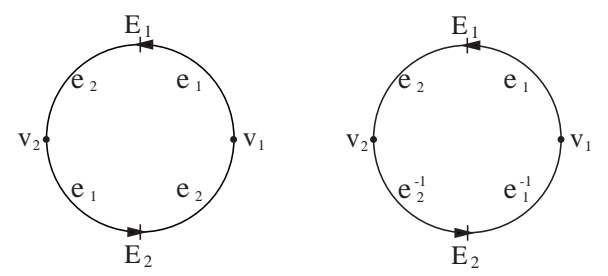

Figure 1. $(X, f)$ and $(X, g)$ with the wrapping rules $\tilde{f}$ and $\tilde{g}$, respectively.

Then $(X, f)$ satisfies all six Axioms, and $(X, g)$ satisfies all Axioms except the Nonfolding Axiom. For $(X, g), g^{2}$ is not locally one-to-one at $\exp \left(\frac{\pi}{2} i\right) \in S^{1}$.

Notation 2.4. Suppose that $(X, f)$ satisfies the Nonfolding Axiom and the Markov Axiom, $\mathcal{E}=\left\{e_{1}, \ldots, e_{n}\right\}$ is the edge set of $X$ with a given direction, and $k$ is a positive integer. For each edge $e_{i} \in \mathcal{E}$, we can give $e_{i}$ the partition $\left\{I_{i, j}^{(k)}\right\}$, $1 \leq j \leq j(i, k)$, for $f^{k}$ such that

(1) the initial point of $I_{i, 1}^{(k)}$ is the initial point of $e_{i}$,

(2) the terminal point of $I_{i, j}^{(k)}$ is the initial point of $I_{i, j+1}^{(k)}$ for $1 \leq j<j(i, k)$,

(3) the terminal point of $I_{i, j(i, k)}^{(k)}$ is the terminal point of $e_{i}$,

(4) $\left.f^{k}\right|_{\operatorname{Int} I_{i, j}^{(k)}}$ is injective, and

(5) $f^{k}\left(I_{i, j}^{(k)}\right)=e_{i, j}^{(k)^{s(i, j, k)}}$ where $e_{i, j}^{(k)} \in \mathcal{E}, s(i, j, k)=1$ if the direction of $f^{k}\left(I_{i, j}^{(k)}\right)$ agree with that of $e_{i, j}^{(k)}$, and $s(i, j, k)=-1$ if the direction of $f^{k}\left(I_{i, j}^{(k)}\right)$ is reverse to that of $e_{i, j}^{(k)}$.

Remarks 2.5. (1) By the Nonfolding Axiom, if $f^{k}\left(I_{i, j}^{(k)}\right)=e_{i, j}^{ \pm 1}$ for $1 \leq j<j(i, k)$, then $f^{k}\left(I_{i, j+1}^{(k)}\right)=e_{i, j+1}$ cannot be $e_{i, j}^{\mp 1}$.

(2) If $(X, f)$ satisfies all six axioms, then there is a positive integer $m$ such that, for every vertex $v$ of $X$ and every integer $k \geq m$, there exist at most two edges $e_{v, k, 1}$ and $e_{v, k, 2}$ such that, for every $I \in\left\{\bar{I}_{i, j}^{(k)} \mid v \in f^{k}\left(I_{i, j}^{(k)}\right)\right\}, f^{k}(I)=e_{v, k, l}^{ \pm 1}$, $l=1$ or 2 .

Definition 2.6. Suppose that $(X, f)$ is a presentation of a branched solenoid, that is, $(X, f)$ satisfies all Axioms except possibly the Flattening Axiom, and $\mathcal{E}$ is the edge set of $X$. Then each edge $e_{i} \in \mathcal{E}$ has the partition $\left\{I_{i, j}^{(1)}\right\}$ for $f$, and we can define an induced map $\tilde{f}: \mathcal{E} \rightarrow \mathcal{E}^{*}$ by

$$
\tilde{f}: e_{i} \mapsto e_{i, 1}^{s(i, 1)} e_{i, 2}^{s(i, 2)} \cdots e_{i, j(i)}^{s(i, j(i))}
$$

where $e_{i, j}^{s(i, j)}=f\left(I_{i, j}^{(1)}\right)$ for $1 \leq j \leq j(i)$. We call $\tilde{f}$ the substitution rule or the wrapping rule associated to $f$.

Examples 2.7. Let $(X, f)$ and $(X, g)$ be given in Examples 2.3. Then $\tilde{f}, \tilde{g}: \mathcal{E}_{X} \rightarrow$ $\mathcal{E}_{X}^{*}$ are given by

$$
\tilde{f}: e_{1} \mapsto e_{1} e_{2}, e_{2} \mapsto e_{1} e_{2}, \text { and } \tilde{g}: e_{1} \mapsto e_{1} e_{2}, e_{2} \mapsto e_{2}^{-1} e_{1}^{-1} .
$$

To establish some notation, we give Figure 1 to represent the presentations $(X, f)$ and $(X, g)$ with the wrapping rules $\tilde{f}$ and $\tilde{g}$, respectively. 


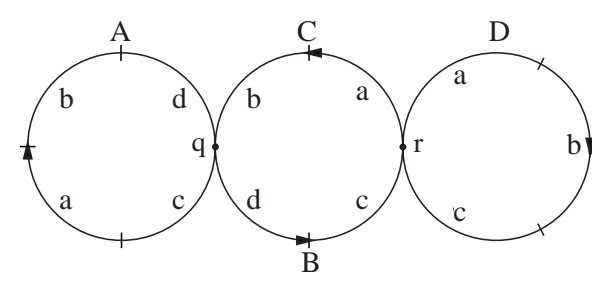

FiguRE 2. $(Y, h)$ with wrapping rule $\tilde{h}$.

Similarly, if $(Y, h)$ is given by Figure 2 then the wrapping rule $\tilde{h}: \mathcal{E}_{Y} \rightarrow \mathcal{E}_{Y}^{*}$ is given by

$$
a \mapsto c a b d, \quad b \mapsto d c, \quad c \mapsto a b, \quad d \mapsto a b c .
$$

Note that the two vertices $q, r$ of $Y$ have $h$-period 2. If $U_{q}$ and $U_{r}$ are sufficiently small neighborhoods of $q$ and $r$, respectively, then $h^{2}\left(U_{q}\right)$ and $h\left(U_{r}\right)$ are intervals. So $(Y, h)$ satisfies the Flattening Axiom.

Lemma 2.8. Suppose that $(X, f)$ satisfies Axioms 4 and 5. Then there is a positive integer $l$ such that, for each edge $e_{i}$ of $X$ and every positive integer $m$, if $\left\{I_{i, 1}^{(l)}, \ldots, I_{i, j(i, l)}^{(l)}\right\}$ and $\left\{I_{i, 1}^{(l m)}, \ldots, I_{i, j(i, l m)}^{(l m)}\right\}$ are partitions of $e_{i}$ for $f^{l}$ and $f^{l m}$, respectively, then $f^{l}\left(I_{i, 1}^{(l)}\right)=f^{l m}\left(I_{i, 1}^{(l m)}\right)$ and $f^{l}\left(I_{i, j(i, l)}^{(l)}\right)=f^{l m}\left(I_{i, j(i, l m)}^{(l m)}\right)$.

Proof. Since $\mathcal{V}$ is a finite set and $f(\mathcal{V}) \subset \mathcal{V}$, every vertex of $X$ is eventually periodic, and there is a positive integer $l_{1}$ such that $f^{l_{1}}(v)=f^{l_{1} m}(v)$ for every $v \in \mathcal{V}$ and every positive integer $m$.

If $e_{i}$ is an edge of $X$ beginning at $v$, and $\left\{I_{i, 1}^{\left(l_{1}\right)}, \ldots, I_{i, j\left(i, l_{1}\right)}^{\left(l_{1}\right)}\right\}$ is the partition of $e_{i}$ for $f^{l_{1}}$, then $f^{l_{1}}\left(I_{i, 1}^{\left(l_{1}\right)}\right)=e_{j}^{s}$ such that $f^{l_{1}}(v) \in e_{j}$. Since $\mathcal{E}$ is a finite set and $f^{l_{1}}(v)$ is a fixed point of $f^{l_{1}}$, there is a positive integer $l_{2}$ such that, for every positive integer $m$, if $e_{j}$ has partitions $\left\{I_{j, 1}^{\left(l_{2}\right)}, \ldots\right\}$ for $f^{l_{2}}$ and $\left\{I_{j, 1}^{\left(l_{2} m\right)}, \ldots\right\}$ for $f^{l_{2} m}$, then $f^{l_{2}}\left(I_{j, 1}^{\left(l_{2}\right)}\right)=f^{l_{2} m}\left(I_{j, 1}^{\left(l_{2} m\right)}\right)$. This shows that $f^{l_{1} l_{2}}\left(I_{i, 1}^{\left(l_{1} l_{2}\right)}\right)=f^{l_{1} l_{2} m}\left(I_{i, 1}^{\left(l_{1} l_{2} m\right)}\right)$ for every positive integer $m$. By the same argument, we can choose a positive integer $l^{\prime}$ for $e_{i}$ such that $f^{l^{\prime}}\left(I_{i, j\left(i, l^{\prime}\right)}^{\left(l^{\prime}\right)}\right)=f^{l^{\prime} m}\left(I_{i, j\left(i, l^{\prime} m\right)}^{\left(l^{\prime} m\right)}\right)$.

Let $\ell_{i}$ be the least common multiple of $l_{1} l_{2}$ and $l^{\prime}$ for each edge $e_{i}$, and $l$ the least common multiple of these $\ell_{i}$ 's. Then we have $f^{l}\left(I_{i, 1}^{(l)}\right)=f^{l m}\left(I_{i, 1}^{(l m)}\right)$ and $f^{l}\left(I_{i, j(i, l)}^{(l)}\right)=f^{l m}\left(I_{i, j(i, l m)}^{(l m)}\right)$ for every positive integer $m$.

Lemma 2.9. Suppose that $(X, f)$ satisfies Axioms 3,4 and 5. Then there exist a positive integer $l$ and $\epsilon>0$ such that, for all $x, y \in X, f^{l}(x) \neq f^{l}(y)$ implies that there is a nonnegative integer $K$ such that $d\left(f^{K}(x), f^{K}(y)\right) \geq \epsilon$.

Proof. For convenience, we will take the metric $d$ on $X$ so that $d(x, y)$ is the length of the shortest path between $x$ and $y$, as explained in Remarks 2.1.

Let $l$ be the integer given in Lemma 2.8 So each edge $e_{i}$ has the partition $\left\{I_{i, j}^{(l)}\right\}$ for $f^{l}: X \rightarrow X$ as in Notation 2.4 Without loss of generality, we suppose each $j(i, l) \geq 3$. Let $\mathcal{P}$ be the collection of the intervals $I_{i, j}^{(l)}$. First choose $\epsilon_{1}>0$ so small that

(i) each interval $I_{i, j}^{(l)}$ has length larger than $2 \epsilon_{1}>0$, 
(ii) if $f^{l}(x)=a \in I_{i, 1}^{(l)}, v$ is the initial point of $e_{i}$, and $d(v, a)<\epsilon_{1}$, then $f^{l}(a) \in$ $I_{i, 1}^{(l)}$, and

(iii) if $f^{l}(y)=b \in I_{i, j(i, l)}^{(l)}, w$ is the terminal point of $e_{i}$, and $d(w, b)<\epsilon_{1}$, then $f^{l}(b) \in I_{i, j(i, l)}^{(l)}$.

Then choose $\epsilon$ such that $0<\epsilon<\epsilon_{1}$ and for every $x$ in the compact set $\underset{\substack{i \\ 1<j<j(i, l)}}{\bigcup} I_{i, j}^{(l)}$

and every $y \in X$, if $0<d(x, y)<\epsilon$, then

(iv) $0<d\left(f^{l}(x), f^{l}(y)\right)<\epsilon_{1}$ and

(v) the interval $\left[f^{l}(x), f^{l}(y)\right]$ contains at most one vertex.

Note that $f^{l}(x) \neq f^{l}(y)$ comes from the Nonfolding Axiom.

If $f^{l}(x)=a \neq b=f^{l}(y)$ and $d(a, b)<\epsilon_{1}$, then $a$ and $b$ lie on the same or adjacent elements of $\mathcal{P}$. So we have two cases:

(1) $a$ and $b$ are end points of an interval of length less than $\epsilon_{1}$ containing a vertex $v$, or

(2) the interval $[a, b]$ of length less than $\epsilon_{1}$ does not contain any vertex of $X$.

For case (1), by the condition (i), $d(a, b)<\epsilon_{1}$ implies that $a$ and $b$ are contained in two different intervals among $I_{i, 1}^{(l)}, I_{i, j(i, l)}^{(l)}, I_{n, 1}^{(l)}$, and $I_{n, j(n, l)}^{(l)}$. For brevity, let us assume $a \in I_{i, 1}$ and $b \in I_{n, 1}$. Then by the condition (ii), $f^{l}(a) \in I_{i, 1}^{(l)}$ and $f^{l}(b) \in I_{n, 1}^{(l)}$. If $v$ is the vertex of $X$ contained in $I_{i, 1}^{(l)} \cap I_{n, 1}^{(l)}$, then $f^{l}$ maps $[v, a]$ into $I_{i, 1}$. So

$$
d\left(f^{l}(a), v\right) \geq c \lambda^{l} \cdot d(a, v)
$$

where $c$ and $\lambda$ are the expansion constants. Similarly $d\left(f^{l}(b), v\right) \geq c \lambda^{l} \cdot d(b, v)$. Let $k$ be the smallest positive integer such that $d\left(v, f^{l k}(a)\right) \geq \epsilon_{1}$ or $d\left(v, f^{l k}(b)\right) \geq \epsilon_{1}$. Then by induction using (ii) and (iii), we have for $0<s \leq k$ that $f^{s l}$ sends $[v, a]$ injectively into $I_{i, 1}$ and $[v, b]$ injectively into $I_{n, 1}$. Therefore we have $d\left(f^{k l}(a), f^{k l}(b)\right) \geq \epsilon_{1}>\epsilon$.

For case (2), let $k$ be the smallest positive integer such that $\left[f^{k l}(a), f^{k l}(b)\right]$ contains a vertex. It follows from the Nonfolding Axiom that $f^{(k-1) l}(a) \neq f^{(k-1) l}(b)$. If there exists $i, 0<i<k$, such that $d\left(f^{i l}(a), f^{i l}(b)\right)>\epsilon$, then we are done, so suppose not. Then $f^{k l}(a)$ and $f^{k l}(b)$ are endpoints of an interval of length less than $\epsilon_{1}$ containing a vertex. Hence we may conclude the proof by applying the argument of case (1).

Definition 2.10 ([10, §3.5]). A homeomorphism $h$ on a metric space $Y$ is expansive if there is an $\epsilon>0$ such that, for all $x \neq y \in Y$, there is an integer $n$ such that

$$
d\left(h^{n}(x), h^{n}(y)\right) \geq \epsilon .
$$

For a solenoid $\bar{X}$ presented by $(X, f)$, define a metric $\bar{d}$ on $\bar{X}$ by

$$
\bar{d}(x, y)=\sum_{i=0}^{\infty} \frac{d\left(x_{i}, y_{i}\right)}{2^{i}}
$$

where $x=\left(x_{0}, x_{1}, \ldots\right), y=\left(y_{0}, y_{1}, \ldots\right) \in \bar{X}$, and $d$ is a metric on $X$ compatible with the topology of $X$ such that $f: X \rightarrow X$ is an expansion with respect to $d$ as in Remarks [2.1. (2). 
Proposition 2.11. If $(X, f)$ satisfies Axioms 3,4 and 5 , then $\bar{f}: \bar{X} \rightarrow \bar{X}$ is expansive.

Proof. For a pair of points $x=\left(x_{0}, x_{1}, \ldots\right) \neq y=\left(y_{0}, y_{1}, \ldots\right) \in \bar{X}$, there is a nonnegative integer $N$ such that $x_{n} \neq y_{n}$ for all $n \geq N$.

Let $l$ and $\epsilon>0$ be given in Lemma [2.9. Then $x_{N+l} \neq y_{N+l}$ and $f^{l}\left(x_{N+l}\right) \neq$ $f^{l}\left(y_{N+l}\right)$ imply that there exists $K \geq 0$ such that $d\left(f^{K}\left(x_{N+l}\right), f^{K}\left(y_{N+l}\right)\right) \geq \epsilon$ by Lemma 2.9. Therefore we have

$$
\bar{d}\left(\bar{f}^{K-N-l}(x), \bar{f}^{K-N-l}(y)\right)=d\left(f^{K}\left(x_{N+l}\right), f^{K}\left(y_{N+l}\right)\right)+\sum_{i>K-N-l} \frac{\left.d\left(x_{i}, y_{i}\right)\right)}{2^{N+l-K+i}}>\epsilon,
$$

and this proves that $\bar{f}: \bar{X} \rightarrow \bar{X}$ is expansive.

Example 2.12. We need the Nonfolding Axiom in Proposition 2.11.

For $f, g: S^{1} \rightarrow S^{1}$ in Examples 2.3 it follows from Proposition 2.11 that $\bar{f}$ is expansive. For $\bar{g}$ with given $\epsilon>0$ and $x=\left(x_{0}, x_{1}, \ldots\right), y=\left(y_{0}, y_{1}, \ldots\right) \in\left(\overline{S^{1}}, \bar{g}\right)$, let

$$
x_{n}=\exp \left(\frac{\pi}{2^{n}} i+\frac{1}{2^{n+2}} \epsilon i\right) \text { and } y_{n}=\exp \left(\frac{\pi}{2^{n}} i-\frac{1}{2^{n+2}} \epsilon i\right) .
$$

Then, for a natural Riemannian metric $d$ on $S^{1}$,

$$
\bar{d}\left(\bar{g}^{k}(x), \bar{g}^{k}(y)\right)=\frac{1}{2^{|k|}} d(x, y)=\frac{1}{2^{|k|}} \epsilon
$$

for every integer $k$, and $\bar{g}$ is not expansive.

SFT covers. We will review the standard construction of a shift of finite type (SFT) cover for one-dimensional branched solenoids.

Suppose that $(X, f)$ is a presentation of a branched solenoid, and $\mathcal{E}=\left\{e_{1}, \ldots, e_{n}\right\}$ is the edge set of $X$. Let $\left\{I_{i, j} \mid 1 \leq i \leq n, 1 \leq j \leq j(i)\right\}$ be the partition of $\mathcal{E}$ for $f$, and $\tilde{f}: \mathcal{E} \rightarrow \mathcal{E}^{*}$ the wrapping rule associated to $f$ given by

$$
\tilde{f}: e_{i}=I_{i, 1} \cdots I_{i, j(i)} \mapsto e_{i, 1}^{s(i, 1)} e_{i, 2}^{s(i, 2)} \cdots e_{i, j(i)}^{s(i, j(i))}
$$

where $e_{i, j}^{s(i, j)}=f\left(I_{i, j}\right)$ and $s(i, j)= \pm 1$ denotes the direction. The adjacency matrix $M$ of $(\mathcal{E}, \tilde{f})$ is given by

$$
M(i, j)=\#\left\{I_{i, l} \mid f\left(I_{i, l}\right)=e_{j}^{ \pm 1}\right\}
$$

We may view $M$ as the adjacency matrix of a directed graph whose vertex set is $\mathcal{E}$ and whose edge set is $\mathcal{A}=\left\{I_{i, j} \mid 1 \leq i \leq n, 1 \leq j \leq j(i)\right\}$, the partition of $\mathcal{E}$ for $f$, where outgoing edges from the 'vertex' $e_{i}$ are named $I_{i, 1}, \ldots, I_{i, j(i)}$.

Now we can give $(\bar{X}, \bar{f})$ a two-sided SFT cover $\left(\Sigma_{X}, \sigma_{X}\right)$ defined from the alphabet $\mathcal{A}$ and the adjacency matrix $M_{X}$. The shift space $\Sigma_{X}$ is the subset of $\mathcal{A}^{\mathbb{Z}}$ whose forbidden blocks are $\left\{I_{i, j} I_{k, l} \mid I_{k, l} \nsubseteq \nsubseteq f\left(I_{i, j}\right)=e_{i, j}^{s(i, j)}\right\}$ from the formula (1). Therefore $\Sigma_{X}$ is a one-step subshift of finite type, and a word $w=I_{a(0)} I_{a(1)} \cdots I_{a(l)}$ is allowed in $\Sigma_{X}$ if and only if $\bigcap_{j=0}^{l} f^{-j}\left(I_{a(j)}\right)$ has nonempty interior.

For each point $w=\cdots I_{a(-1)} I_{a(0)} I_{a(1)} \cdots \in \Sigma_{X}$ and the canonical projection map onto the zeroth coordinate $\pi: \bar{X} \rightarrow X$, there is a unique corresponding point

$$
x_{w}=\left(x_{0}, x_{1}, \ldots\right)=\bigcap_{j=-\infty}^{\infty} \overline{\bar{f}^{-j}\left(\pi^{-1}\left(I_{a(j)}\right)\right)} \in \bar{X}
$$


such that $x_{i} \in I_{a(-i)}$ and $f^{i}\left(x_{0}\right) \in I_{a(i)}$ for $i \geq 0$. Hence there is a well-defined semiconjugacy $p: \Sigma_{X} \rightarrow \bar{X}$ defined by $w \mapsto x_{w}$. It is not difficult to check $\bar{f} \circ p=$ $p \circ \sigma_{X}([9, \S 6.5])$.

Proposition 2.13 ([4, §3.D]). Suppose that $(X, f)$ satisfies all six axioms except possibly the Flattening Axiom. Let $p$ and $\Sigma_{X}$ be as above. Then

(1) $p: \Sigma_{X} \rightarrow \bar{X}$ is finite-to-one.

(2) $p$ is one-to-one on $\Sigma_{X} \backslash \bigcup_{m=0}^{\infty} p^{-1} \circ \pi^{-1} \circ f^{-m-1}(\mathcal{V})$ where $\pi: \bar{X} \rightarrow X$ is the projection map to the zeroth coordinate space and $\mathcal{V}$ is the vertex set of $X$.

(3) $(\bar{X}, \bar{f})$ and $\left(\Sigma_{X}, \sigma_{X}\right)$ have the same entropy.

Lemma $2.14([15,1.6])$. If $(X, f)$ satisfies all six axioms, and $I$ is an interval in $X$, then $X \subset f^{m}(I)$ for some $m \geq 0$.

We remark that the proof of $[15,1.6]$ still works in our topological setting. Then for all $I_{i, j}, I_{k, l} \in \mathcal{A}, I_{k, l} \subset f^{m}\left(I_{i, j}\right)$ for some positive integer $m$, and we have the following proposition.

Proposition 2.15. If $(X, f)$ satisfies all six axioms, then $\left(\Sigma_{X}, \sigma_{X}\right)$ is a mixing SFT.

Examples 2.16. Let $(X, f)$ and $(Y, h)$ be as in Examples 2.7. Recall that $\tilde{f}: \mathcal{E}_{X} \rightarrow$ $\mathcal{E}_{X}^{*}$ and $\tilde{h}: \mathcal{E}_{Y} \rightarrow \mathcal{E}_{Y}^{*}$ are given by

$$
\begin{aligned}
& \tilde{f}: e_{1} \mapsto e_{1} e_{2}, e_{2} \mapsto e_{1} e_{2}, \\
& \tilde{h}: a \mapsto c a b d, b \mapsto d c, \text { and } c \mapsto a b c, d \mapsto a b .
\end{aligned}
$$

The SFT covers of $(\bar{X}, \bar{f})$ and $(\bar{Y}, \bar{h})$ are given by the following matrices:

$$
M_{X}=\left(\begin{array}{ll}
1 & 1 \\
1 & 1
\end{array}\right) \text { and } M_{Y}=\left(\begin{array}{cccc}
1 & 1 & 1 & 1 \\
0 & 0 & 1 & 1 \\
1 & 1 & 0 & 0 \\
1 & 1 & 1 & 0
\end{array}\right)
$$

Example 2.17. We need the Flattening Axiom in Lemma 2.14 and Proposition 2.15. Let $Z$ be a wedge product of two circles $a$ and $b$, and $g: Z \rightarrow Z$ given by $a \mapsto a a$ and $b \mapsto b b$. Then $(Z, g)$ does not satisfy the Flattening Axiom, and the adjacency matrix $M_{Z}$ for $(\mathcal{E}, \tilde{g})$ is $M_{Z}=\left(\begin{array}{ll}2 & 0 \\ 0 & 2\end{array}\right)$. So $\left(\Sigma_{Z}, \sigma_{Z}\right)$ is not irreducible.

\section{Shift Equivalence}

We define shift equivalence of directed graphs with graph maps, and show that conjugacy of the shift maps on branched solenoids is equivalent to shift equivalence of their presentations and that certain conjugacies of shift maps lift uniquely to conjugacies of associated SFT covers.

Definition 3.1. Suppose that $X$ and $Y$ are directed graphs, and that $f: X \rightarrow X$ and $g: Y \rightarrow Y$ are graph maps. We say that $f$ and $g$ are shift equivalent with lag $m$ if there are continuous maps $r: X \rightarrow Y, s: Y \rightarrow X$ and a positive integer $m$ such that

$$
r \circ f=g \circ r, \quad f \circ s=s \circ g, \quad f^{m}=s \circ r, \quad g^{m}=r \circ s .
$$


If $(X, f)$ and $(Y, g)$ are shift equivalent with lag $m$ by continuous maps $r: X \rightarrow Y$ and $s: Y \rightarrow X$, then define induced maps $\bar{r}: \bar{X} \rightarrow \bar{Y}$ and $\bar{s}: \bar{Y} \rightarrow \bar{X}$ by

$$
\begin{aligned}
& \bar{r}:\left(x_{0}, \ldots, x_{m}, x_{m+1}, \ldots\right) \mapsto\left(r\left(x_{m}\right), r\left(x_{m+1}\right), \ldots\right), \\
& \bar{s}:\left(y_{0}, y_{1}, y_{2}, \ldots\right) \mapsto\left(s\left(y_{0}\right), s\left(y_{1}\right), s\left(y_{2}\right), \ldots\right) .
\end{aligned}
$$

We can easily check that $\bar{r}$ and $\bar{s}$ are topological conjugacies of $\bar{f}$ and $\bar{g}$ such that $\bar{s} \circ \bar{r}=I d$ on $\bar{X}$ and $\bar{r} \circ \bar{s}=I d$ on $\bar{Y}$.

Remark 3.2. It is possible that the shift equivalence map $r: X \rightarrow Y$ is not a graph map, that is, a vertex of $X$ may not be mapped to a vertex of $Y$.

Lemma 3.3. Suppose that $(X, f)$ and $(Y, g)$ satisfy the Markov Axiom, and they are shift equivalent to each other with lagm by continuous maps $r: X \rightarrow Y$ and $s: Y \rightarrow X$. Then the vertex sets $\mathcal{V}_{X}$ and $\mathcal{V}_{Y}$ of $X$ and $Y$, respectively, can be enlarged to $\mathcal{V}_{X}^{\prime}$ and $\mathcal{V}_{Y}^{\prime}$, respectively, so that

$$
f\left(\mathcal{V}_{X}^{\prime}\right) \subset \mathcal{V}_{X}^{\prime}, g\left(\mathcal{V}_{Y}^{\prime}\right) \subset \mathcal{V}_{Y}^{\prime}, r\left(\mathcal{V}_{X}^{\prime}\right) \subset \mathcal{V}_{Y}^{\prime} \text {, and } s\left(\mathcal{V}_{Y}^{\prime}\right) \subset \mathcal{V}_{X}^{\prime}
$$

Proof. For the vertex sets $\mathcal{V}_{X}$ and $\mathcal{V}_{Y}$, let $\mathcal{V}_{Y}^{\prime}=\mathcal{V}_{Y} \cup r\left(\mathcal{V}_{X}\right)$ be the set of enlarged vertices in $Y$ and $\mathcal{V}_{X}^{\prime}=\mathcal{V}_{X} \cup s\left(\mathcal{V}_{Y}^{\prime}\right)$. Then

$$
\begin{aligned}
& g\left(\mathcal{V}_{Y}^{\prime}\right) \subset g\left(\mathcal{V}_{Y}\right) \cup g \circ r\left(\mathcal{V}_{X}\right) \subset \mathcal{V}_{Y} \cup r \circ f\left(\mathcal{V}_{X}\right) \subset \mathcal{V}_{Y} \cup r\left(\mathcal{V}_{X}\right)=\mathcal{V}_{Y}^{\prime}, \\
& f\left(\mathcal{V}_{X}^{\prime}\right) \subset f\left(\mathcal{V}_{X}\right) \cup f \circ s\left(\mathcal{V}_{Y}^{\prime}\right) \subset \mathcal{V}_{X} \cup s \circ g\left(\mathcal{V}_{Y}^{\prime}\right) \subset \mathcal{V}_{X} \cup s\left(\mathcal{V}_{Y}^{\prime}\right)=\mathcal{V}_{X}^{\prime}, \\
& r\left(\mathcal{V}_{X}^{\prime}\right)=r\left(\mathcal{V}_{X}\right) \cup r \circ s\left(\mathcal{V}_{Y}^{\prime}\right)=r\left(\mathcal{V}_{X}\right) \cup g^{m}\left(\mathcal{V}_{Y}^{\prime}\right) \subseteq \mathcal{V}_{Y}^{\prime} \text { and } \\
& s\left(\mathcal{V}_{Y}^{\prime}\right)=s\left(\mathcal{V}_{Y}\right) \cup f^{m}\left(\mathcal{V}_{X}\right) \subseteq \mathcal{V}_{X}^{\prime}
\end{aligned}
$$

prove the lemma.

Lemma 3.4 (Ladder Lemma). Suppose that $(X, f)$ and $(Y, g)$ satisfy all axioms except possibly the Flattening Axiom, and that $\phi: \bar{X} \rightarrow \bar{Y}$ is a continuous map such that $\bar{g} \circ \phi=\phi \circ \bar{f}$. Then there is a continuous map $r: X \rightarrow Y$ and a nonnegative integer $n$ such that $g \circ r=r \circ f$ and $\phi\left(x_{0}, x_{1}, \ldots\right)=\left(r\left(x_{n}\right), r\left(x_{n+1}\right), \ldots\right)$.

Remark 3.5. Williams ([15, §3]) proved the Ladder Lemma under the hypotheses that $f$ and $g$ are nonwandering expansions which are immersions of differentiable branched one-dimensional manifolds and satisfy the Flattening Axiom. Our assumptions are weaker than his conditions as we don't need the Flattening Axiom, but the ideas of the proof are essentially those given by Williams. The additional complication of our setting is addressed in Lemma 2.9

Let $X_{i}$ and $Y_{i}$ be the $i$ th coordinate spaces of $\bar{X}$ and $\bar{Y}$, respectively, and $\pi_{i}$ the projection map from the branched solenoids onto their $i$ th coordinate spaces.

Lemma 3.6. There is a positive integer $n$ such that, for $a, b \in \bar{X}$, if $\pi_{n}(a)=\pi_{n}(b)$, then $\pi_{0} \circ \phi(a)=\pi_{0} \circ \phi(b)$.

Proof. By Lemma 2.9] we can choose $\epsilon>0$ and $l \in \mathbb{N}$ such that, for all $x, y \in Y$, if $g^{l}(x) \neq g^{l}(y)$, then there exists a nonnegative integer $K$ such that

$$
d\left(g^{K}(x), g^{K}(y)\right) \geq \epsilon .
$$

Choose a nonnegative integer $k$ and $\delta>0$ such that, for $a=\left(a_{0}, a_{1}, \ldots\right)$ and $b=$ $\left(b_{0}, b_{1}, \ldots\right) \in \bar{X}, a_{k}=b_{k}$ implies $d(a, b)<\delta$, and $d(a, b)<\delta$ implies $d(\phi(a), \phi(b))$ $<\epsilon$. 
Now suppose $a_{k}=b_{k}$. From $d\left(\bar{f}^{m}(a), \bar{f}^{m}(b)\right) \leq d(a, b)<\delta$ for every nonnegative integer $m$, we have

$$
d\left(\phi \circ \bar{f}^{m}(a), \phi \circ \bar{f}^{m}(b)\right)=d\left(\bar{g}^{m} \circ \phi(a), \bar{g}^{m} \circ \phi(b)\right)<\epsilon,
$$

and if $x_{0}=\pi_{0} \circ \phi(a)$ and $y_{0}=\pi_{0} \circ \phi(b)$, then for every nonnegative integer $m$

$$
d\left(g^{m}\left(x_{0}\right), g^{m}\left(y_{0}\right)\right)<\epsilon .
$$

Therefore we have that $a_{k}=b_{k}$ implies $g^{l} \circ \pi_{0} \circ \phi(a)=g^{l} \circ \pi_{0} \circ \phi(b)$, and equivalently $a_{k+l}=b_{k+l}$ implies $\pi_{0} \circ \phi(a)=\pi_{0} \circ \phi(b)$.

Proof of Ladder Lemma. Let $n$ be as in Lemma [3.6] Define $r_{k}: X \rightarrow Y$ by $r_{k}=$ $\pi_{k} \circ \phi \circ \pi_{n+k}^{-1}$. Then $r_{k}$ is well-defined by our choice of $n$. Now show that $r_{k}=r_{0}$ for every positive integer $k$. For $x=\left(x_{0}, \ldots, x_{n}, \ldots\right) \in \bar{X}$,

$$
\begin{aligned}
r_{k}\left(x_{n}\right) & =\pi_{k} \circ \phi \circ \pi_{n+k}^{-1}\left(x_{n}\right)=\pi_{k} \circ \phi \circ \bar{f}^{k}(x) \\
& =\pi_{k} \circ \bar{g}^{k} \circ \phi(x)=\pi_{0} \circ \phi(x)=\pi_{0} \circ \phi \circ \pi_{n}^{-1}\left(x_{n}\right) \\
& =r_{0}\left(x_{n}\right) .
\end{aligned}
$$

To show the continuity of $r=r_{0}$, let $\delta>0$ and $\epsilon>0$ be as in Lemma 3.6. and choose $\delta^{\prime}>0$ such that if $a_{n}, b_{n}$ are elements in $X$ with $d\left(a_{n}, b_{n}\right)<\delta^{\prime}$, then there exist $a, b \in \bar{X}$ with $\pi_{n}(a)=a_{n}$ and $\pi_{n}(b)=b_{n}$ such that $d(a, b)<\delta$. Then we have

$$
d\left(a_{n}, b_{n}\right)<\delta^{\prime} \Longrightarrow d\left(\pi_{0} \circ \phi(a), \pi_{0} \circ \phi(b)\right)=d\left(r\left(a_{n}\right), r\left(b_{n}\right)\right)<\epsilon,
$$

and $r: X \rightarrow Y$ is continuous. That $\phi\left(x_{0}, \ldots, x_{n}, x_{n+1}, \ldots\right)=\left(r\left(x_{n}\right), r\left(x_{n+1}\right), \ldots\right)$ is trivial by the construction of $r: X \rightarrow Y$.

The proof of the following proposition is easy from the Ladder Lemma. So we omit the proof.

Proposition 3.7 ([15, Theorem 3.3]). Suppose that $(X, f)$ and $(Y, g)$ satisfy all axioms except possibly the Flattening Axiom. Then $\phi: \bar{X} \rightarrow \bar{Y}$ is a topological conjugacy if and only if there exists a shift equivalence $(r, s)$ of $f$ and $g$ such that $\phi=\bar{r}$.

Let $\left(\Sigma_{X}, \sigma_{X}\right)$ and $\left(\Sigma_{Y}, \sigma_{Y}\right)$ be the SFT covers of $(\bar{X}, \bar{f})$ and $(\bar{Y}, \bar{g})$ defined by $\left(\mathcal{E}_{X}, \tilde{f}\right)$ and $\left(\mathcal{E}_{Y}, \tilde{g}\right)$, respectively, as in 2 .

Theorem 3.8. Suppose that $(X, f)$ and $(Y, g)$ satisfy Axioms 3, 4, and 5, and they are shift equivalent to each other with lagm by graph maps $r$ and $s$. Then the conjugacy $\bar{r}: \bar{X} \rightarrow \bar{Y}$ lifts to a unique conjugacy $\tilde{r}$ of $\left(\Sigma_{X}, \sigma_{X}\right)$ and $\left(\Sigma_{Y}, \sigma_{Y}\right)$.

Proof. We will define a sliding block code $\phi_{r}: \Sigma_{X} \rightarrow \Sigma_{Y}$ induced by $r$, and show that $\phi_{r}$ is the required conjugacy $\tilde{r}$.

Let $\mathcal{E}_{X}$ and $\mathcal{E}_{Y}$ denote the edge sets of $X$ and $Y$, respectively, $\mathcal{A}_{X}=\left\{I_{i, j}\right\}$ and $\mathcal{A}_{Y}=\left\{J_{k, l}\right\}$ the alphabets of $\left(\Sigma_{X}, \sigma_{X}\right)$ and $\left(\Sigma_{Y}, \sigma_{Y}\right)$, respectively, and $p_{X}: \Sigma_{X} \rightarrow$ $\bar{X}$ and $p_{Y}: \Sigma_{Y} \rightarrow \bar{Y}$ the semiconjugacies. Then $I_{i, j}$ is a subset of $e_{i} \in \mathcal{E}_{X}$ such that $f\left(I_{i, j}\right)=e_{i, j} \in \mathcal{E}_{X}$ (ignoring the direction). Note that if $a=\cdots I_{a(-1)} I_{a(0)} I_{a(1)} \cdots \in$ $\Sigma_{X}, x_{a}=\left(x_{0}, x_{1}, \ldots\right)=p_{X}(a) \in \bar{X}$, and $I_{a(i)} \subset e_{a_{i}}$, then $x_{i} \in I_{a(-i)}$ and $f^{i}\left(x_{0}\right) \in I_{a(i)}$ for every nonnegative integer $i$.

Let $\mathcal{C}_{X}=\left\{a \in \Sigma_{X} \mid p_{X}^{-1} \circ p_{X}(a)=\{a\}\right\}$ and $\mathcal{C}_{Y}=\left\{b \in \Sigma_{Y} \mid p_{Y}^{-1} \circ p_{Y}(b)=\{b\}\right\}$. Then by Proposition 2.13 $\mathcal{C}_{X}$ and $\mathcal{C}_{Y}$ are dense in $\Sigma_{X}$ and $\Sigma_{Y}$, respectively. 
Step 1. Show that $\bar{r} \circ p_{X}\left(\mathcal{C}_{X}\right)=p_{Y}\left(\mathcal{C}_{Y}\right)$.

By Proposition[2.13, $a \in \mathcal{C}_{X}$ if and only if $\pi_{0} \circ p_{X}(a) \notin \bigcup_{n=0}^{\infty} f^{-n-1}\left(\mathcal{V}_{X}\right)$ where $\pi_{i}$ is the projection map from the branched solenoids to their $i$ th coordinate spaces. So we have $\bar{f}^{m} \circ p_{X}\left(\mathcal{C}_{X}\right)=p_{X}\left(\mathcal{C}_{X}\right)$.

If $\bar{r} \circ p_{X}(a) \notin p_{Y}\left(\mathcal{C}_{Y}\right)$ for some $a \in \mathcal{C}_{X}$, then $r \circ \pi_{m} \circ p_{X}(a) \in \bigcup_{n=0}^{\infty} g^{-n-1}\left(\mathcal{V}_{Y}\right)$ and

$$
g^{n+1} \circ r \circ \pi_{m} \circ p_{X}(a)=r \circ f^{n+1} \circ \pi_{m} \circ p_{X}(a) \in \mathcal{V}_{Y}
$$

for some $n \geq 0$. Since the shift equivalence maps $r$ and $s$ are graph maps by Lemma 3.3 and $s \circ r=f^{m}$, we have

$$
s \circ r \circ f^{n+1} \circ \pi_{m} \circ p_{X}(a)=f^{n+m+1} \circ \pi_{m} \circ p_{X}(a)=f^{n+1} \circ \pi_{0} \circ p_{X}(a) \in \mathcal{V}_{X},
$$

a contradiction. Hence we have $\bar{r} \circ p_{X}\left(\mathcal{C}_{X}\right) \subset p_{Y}\left(\mathcal{C}_{Y}\right)$. By the same argument, we can show that $\bar{s} \circ p_{Y}\left(\mathcal{C}_{Y}\right) \subset p_{X}\left(\mathcal{C}_{X}\right)$. Then $\bar{r} \circ \bar{s}=\bar{g}^{m}$ and $\bar{g}^{m}\left(\mathcal{C}_{Y}\right)=\mathcal{C}_{Y}$ imply that

$$
p_{Y}\left(\mathcal{C}_{Y}\right)=\bar{r} \circ \bar{s} \circ p_{Y}\left(\mathcal{C}_{Y}\right) \subset \bar{r} \circ p_{X}\left(\mathcal{C}_{X}\right)
$$

Therefore we have $\bar{r} \circ p_{X}\left(\mathcal{C}_{X}\right)=p_{Y}\left(\mathcal{C}_{Y}\right)$.

Now we have a well-defined bijective map $\left.\left(p_{Y} \mid \mathcal{C}_{Y}\right)^{-1} \circ \bar{r} \circ p_{X}\right|_{\mathcal{C}_{X}}: \mathcal{C}_{X} \rightarrow \mathcal{C}_{Y}$. This map will define $\tilde{r}$ on $\mathcal{C}_{X}$.

Step 2. Find a block map $\Phi_{r}: \mathcal{B}_{m+1}\left(\mathcal{C}_{X}\right) \rightarrow \mathcal{A}_{Y}$ where $\mathcal{B}_{m+1}\left(\mathcal{C}_{X}\right)$ is the set of all $(m+1)$-blocks in $\mathcal{C}_{X}$ such that for every $a=\cdots I_{a(-m+i)} \cdots I_{a(-1+i)} I_{a(i)} \cdots \in \mathcal{C}_{X}$ $\left.\left(p_{Y} \mid \mathcal{C}_{Y}\right)^{-1} \circ \bar{r} \circ p_{X}\right|_{\mathcal{C}_{X}}(a)_{i}=\Phi_{r}\left(I_{a(-m+i)} \cdots I_{a(i)}\right)$.

For $a=\cdots I_{a(-m)} \cdots I_{a(-1)} I_{a(0)} \cdots \in \mathcal{C}_{X}$, let $x_{a}=\left(x_{0}, x_{1}, \ldots\right)=p_{X}(a) \in \bar{X}$ and $y_{a}=\left(y_{0}, y_{1}, \ldots\right)=\bar{r}\left(x_{a}\right) \in p_{Y}\left(\mathcal{C}_{Y}\right)$. Then $x_{i} \in I_{a(-i)} \subset e_{a(-i)}, y_{i}=r\left(x_{i+m}\right)$, and there exists a unique $\alpha=\cdots J_{\alpha(-1)} J_{\alpha(0)} \cdots \in \mathcal{C}_{Y}$ such that

$$
\left.\left(p_{Y} \mid \mathcal{C}_{Y}\right)^{-1} \circ \bar{r} \circ p_{X}\right|_{\mathcal{C}_{X}}(a)=\alpha \text { and } p_{Y}(\alpha)=y_{a}
$$

Let $\left\{I_{e, j}^{(k)}\right\}$ be the partition of $e=e_{a(-m)} \in \mathcal{E}_{X}$ for $f^{k}, 1 \leq k \leq m+1$. Then each $I_{e, j}^{(k)}$ is contained in a unique $I_{e, j^{\prime}}^{(k-1)}$, and we have a unique descending sequence

$$
e \supset I_{a(-m)}=I_{e, u(1)}^{(1)} \supset I_{e, u(2)}^{(2)} \supset \cdots \supset I_{e, u(m+1)}^{(m+1)}
$$

such that $f^{k}\left(I_{e, u(k)}^{(k)}\right)=e_{a(-m+k)} \in \mathcal{E}_{X}, f^{k}\left(I_{e, u(k+1)}^{(k+1)}\right)=I_{a(-m+k)} \subset e_{a(-m+k)}$, and $x_{m} \in I_{e, u(m+1)}^{(m+1)}$.

Since the shift equivalence maps $r$ and $s$ are graph maps and $s \circ r=f^{m}$, $f^{m}\left(I_{e, u(m)}^{(m)}\right)=e_{a(0)}$ and $f^{m+1}\left(I_{e, u(m+1)}^{(m+1)}\right)=e_{a(1)}$ imply that $r\left(I_{e, u(m)}^{(m)}\right)$ is contained in a unique edge $\epsilon$ in $Y$ and that $r\left(I_{e, u(m+1)}^{(m+1)}\right)$ is contained in a unique path $J$ such that $J \subset \epsilon$ and $g(J) \in \mathcal{E}_{Y}$. Define a block map $\Phi_{r}: \mathcal{B}_{m+1}\left(\mathcal{C}_{X}\right) \rightarrow \mathcal{A}_{Y}$ by

$$
I_{a(-m)} \cdots I_{a(-1)} I_{a(0)} \mapsto J .
$$

Then the sliding block code $\phi_{r}$ induced by $\Phi_{r}$ maps $a$ to $\beta=\cdots J_{\beta(0)} J_{\beta(1)} \cdots$ with $J_{\beta(i)}=\Phi_{r}\left(I_{a(-m+i)} \cdots I_{a(i)}\right)$.

To prove that $\phi_{r}=\left.\left(p_{Y} \mid \mathcal{C}_{Y}\right)^{-1} \circ \bar{r} \circ p_{X}\right|_{\mathcal{C}_{X}}$, we need only show that $p_{Y}(\beta)=y_{a}$, that is, $y_{i}=r\left(x_{i+m}\right) \in J_{\beta(-i)}$. From the descending sequence for $x_{i+m}$

$$
e_{a(-i-m)} \supset I_{a(-i-m)} \supset I_{a(-i-m), u(2)}^{(2)} \supset \cdots \supset I_{a(-i-m), u(m+1)}^{(m+1)} \ni x_{i+m},
$$


$J_{\beta(-i)}$ is the unique path in $Y$ such that $r\left(I_{a(-i-m), u(m+1)}^{(m+1)}\right) \subset J_{\beta(-i)}$ and $g\left(J_{\beta(-i)}\right) \in$ $\mathcal{E}_{Y}$. So $y_{i}=r\left(x_{i+m}\right)$ is contained in $J_{\beta(-i)}$, and $p_{Y}(\beta)=y_{a}$. Therefore $\phi_{r}(a)=$ $\beta=\alpha=\left.\left(\left.p_{Y}\right|_{\mathcal{C}_{Y}}\right)^{-1} \circ \bar{r} \circ p_{X}\right|_{\mathcal{C}_{X}}(a)$ by the definition of $\mathcal{C}_{Y}$, and this proves $\left.\phi_{r}\right|_{\mathcal{C}_{X}}=$ $\left.\left(\left.p_{Y}\right|_{\mathcal{C}_{Y}}\right)^{-1} \circ \bar{r} \circ p_{X}\right|_{\mathcal{C}_{X}}$.

Step 3. Define a block map $\Psi_{s}: \mathcal{B}_{m+1}\left(\mathcal{C}_{Y}\right) \rightarrow \mathcal{A}_{X}$ with $\psi_{s}$ defined on $\mathcal{C}_{Y}$ by $\psi_{s}(\alpha)_{i}=\Psi_{s}\left(J_{\alpha(i)} \cdots J_{\alpha(i+m)}\right)$ such that $\psi_{s} \circ \phi_{r}=I d$ on $\mathcal{C}_{X}$.

We define $\Psi_{s}$ from $s: Y \rightarrow X$ just as we defined $\Phi_{r}$ from $r$ in Step 2. For $\alpha=\cdots J_{\alpha(-i)} J_{\alpha(-i+1)} \cdots \in \mathcal{C}_{Y}$ with $J_{\alpha(-i)} \subset \epsilon$, let $\left\{J_{\epsilon, l(k)}^{(k)}\right\}$ be the partition of $\epsilon \in \mathcal{E}_{Y}$ for $g^{k}, 1 \leq k \leq m+1$, so that $g^{k}\left(J_{\epsilon, l(k)}^{(k)}\right)=\epsilon_{\alpha(-i+k)} \in \mathcal{E}_{Y}$ and $g^{k}\left(J_{\epsilon, l(k+1)}^{(k+1)}\right)=$ $J_{\alpha(-i+k)} \subset \epsilon_{\alpha(-i+k)}$. Then we have a unique descending sequence

$$
\epsilon \supset J_{\alpha(-i)}=J_{\epsilon, v(1)}^{(1)} \supset \cdots \supset J_{\epsilon, v(m+1)}^{(m+1)}
$$

such that, for $y=p_{Y}(\alpha)$, we have $y_{i} \in J_{\epsilon, v(m+1)}^{(m+1)}$ and $s\left(J_{\epsilon, v(m)}^{(m)}\right)$ is contained in a unique path $I$ in $X$ such that $f(I) \in \mathcal{E}_{X}$. We define $\Psi_{s}: \mathcal{B}_{m+1}\left(\mathcal{C}_{Y}\right) \rightarrow \mathcal{A}_{X}$ by

$$
J_{\alpha(-i)} \cdots J_{\alpha(-i+m)} \mapsto I .
$$

Let $\left.p_{X}\right|_{\mathcal{C}_{X}}(a)=x, \bar{r}(x)=y$, and $\left(\left.p_{Y}\right|_{\mathcal{C}_{Y}}\right)^{-1}(y)=\alpha$. If $x_{i+m} \in I_{a(-i-m), u(m+1)}^{(m+1)}$ and $y_{i} \in J_{\epsilon, v(m+1)}^{(m+1)}$ as in the equations (2) and (3), then we have $J_{\epsilon, v(m+1)}^{(m+1)} \subset$ $r\left(I_{a(-i-m), j(m+1)}^{(m+1)}\right)$ and

$$
s\left(J_{\alpha(-i), v(m+1)}^{(m+1)}\right) \subset s \circ r\left(I_{a(-i-m), j(m+1)}^{(m+1)}\right)=I_{a(-i)} \in \mathcal{A}_{X} .
$$

Therefore $\psi_{s} \circ \phi_{r}: \mathcal{C}_{X} \rightarrow \mathcal{C}_{X}$ is the sliding block code with memory $m$ and anticipation $m$ induced by a block map defined by

$$
I_{a(-i-m)} \cdots I_{a(-i)} \cdots I_{a(-i+m)} \mapsto I_{a(-i)},
$$

and $\psi_{s} \circ \phi_{r}=I d$ on $\mathcal{C}_{X}$.

Step 4. Deduce that $\phi_{r}$ gives the required conjugacy $\tilde{r}$.

Because $\phi_{r}$ maps $\mathcal{C}_{X}$ onto $\mathcal{C}_{Y}$, and these sets are dense in $\Sigma_{X}$ and $\Sigma_{Y}$, it follows that $\phi_{r}$ maps $\Sigma_{X}$ onto $\Sigma_{Y}$. Similarly $\psi_{s}$ maps $\Sigma_{Y}$ onto $\Sigma_{X}$. Since the continuous maps $\left.I d\right|_{\Sigma_{X}}$ and $\psi_{s} \circ \phi_{r}$ agree on the dense set $\mathcal{C}_{X}$, we have $\left.I d\right|_{\Sigma_{X}}=\psi_{s} \circ \phi_{r}$ on $\Sigma_{X}$, and so $\phi_{r}$ is a conjugacy.

That $\phi_{r}$ is a lift of $\bar{r}$ follows because $p_{Y} \circ \phi_{r}=\bar{r} \circ p_{X}$ on the dense set $\mathcal{C}_{X}$. The lifting is unique for it is uniquely determined on the dense set $\mathcal{C}_{X}$.

Remark 3.9. It is necessary to assume the shift equivalence by graph maps. See Examples 4.8 and 5.1 .

\section{Graph algorithm}

Suppose that $(X, f)$ is a presentation of a solenoid satisfying all six axioms. Given a finite subset $\mathcal{O}$ of $X$ such that $f(\mathcal{O})=\mathcal{O}$, we will construct a new presentation $\left(X_{\mathcal{O}}, f_{\mathcal{O}}\right)$ such that $\left(\bar{X}_{\mathcal{O}}, \bar{f}_{\mathcal{O}}\right)$ is topologically conjugate to $(\bar{X}, \bar{f})$. For this purpose, we will give a graph algorithm which takes the given presentation $(X, f)$ and $\mathcal{O}$ to produce a new presentation $\left(X_{\mathcal{O}}, f_{\mathcal{O}}\right)$ and shift equivalence maps $\rho_{\mathcal{O}}:\left(X_{\mathcal{O}}, f_{\mathcal{O}}\right) \rightarrow$ $(X, f)$ and $\psi_{\mathcal{O}}:(X, f) \rightarrow\left(X_{\mathcal{O}}, f_{\mathcal{O}}\right)$. 
Notation 4.1. By a path or directed path $l$, we mean an equivalence class of locally one-to-one continuous maps $\gamma:[0,1] \rightarrow X$ where $\gamma_{1} \sim \gamma_{2}$ if and only if there is an order-preserving homeomorphism $h_{1,2}:[0,1] \rightarrow[0,1]$ such that $\gamma_{1}(t)=\gamma_{2} \circ h_{1,2}(t)$ for all $t \in[0,1]$. So a path $l=[\gamma]$ has the initial point $\gamma(0)$ and the terminal point $\gamma(1)$. By abuse of notation, when we say that a path contains a set, we mean that the image of the path contains the set.

Let $\mathcal{P}$ be the set of directed paths $l \subset X$ such that the boundary points of $l$ are contained in $\mathcal{O}$, and the interior of $l$ does not contain any point of $\mathcal{O}$. Let $l$ be a directed path in $\mathcal{P}$. Since $f(\mathcal{O})=\mathcal{O}$, if we consider $f(l)$ as a continuous map $L:[0,1] \rightarrow X$, then the interval $[0,1]$ can be represented as a union of subintervals $\left[0, a_{1}\right] \cup\left[a_{1}, a_{2}\right] \cup \cdots \cup\left[a_{n-1}, 1\right]$ such that $L\left(a_{i}\right) \in \mathcal{O}$ and $L\left(\left(a_{i-1}, a_{i}\right)\right) \cap \mathcal{O}=\emptyset$ for every $0<i<n$. Hence for the collection of $\left[a_{i-1}, a_{i}\right]$ such that $L\left(\left[a_{i-1}, a_{i}\right]\right)=l_{i} \in \mathcal{P}$, $f: X \rightarrow X$ induces a wrapping rule $\tilde{f}_{\mathcal{P}}: \mathcal{P} \rightarrow \mathcal{P}^{*}$ defined by $l \mapsto l_{1^{\prime}} \cdots l_{n^{\prime}}$. We write $l_{i} \in \tilde{f}(l)$ if $l_{i} \in \mathcal{P}$ is one of these factors of $\tilde{f}(l)$. (We remark that some factors of $\tilde{f}(l)$ may not be paths, because $\tilde{f}(l)$ need not be locally one-to-one.)

Our first task is to find a minimal set of directed paths $\mathcal{P}_{\mathcal{O}}$ such that $\mathcal{P}_{\mathcal{O}}$ is a finite subset of $\mathcal{P}, X \subset \bigcup_{l \in \mathcal{P}_{\mathcal{O}}} l, \tilde{f}(l) \in \mathcal{P}_{\mathcal{O}}^{*}$ for every $l \in \mathcal{P}_{\mathcal{O}}$, and there exists a positive integer $k$ such that $l_{1} \in \tilde{f}^{k}\left(l_{2}\right)$ for all $l_{1}, l_{2} \in \mathcal{P}_{\mathcal{O}}$.

Let us denote $\mathcal{I}_{m}$ as the set of directed paths whose boundary points are contained in $f^{-m}(\mathcal{O})$, and whose interior does not have any point in $f^{-m}(\mathcal{O})$. Since we assumed all six axioms, there is a positive integer $n$ such that $f^{n}(e) \supseteq X$ for every edge $e$ of $X$ from Lemma 2.14. So each edge $e$ contains at least one point of $f^{-n}(\mathcal{O})$, and $\mathcal{I}_{m}$ is a finite set for $m \geq n$.

We have the following lemma from the Flattening Axiom.

Lemma 4.2. There exists a positive integer $N \geq n$ such that the interior of each edge contains at least one point of $f^{-N}(\mathcal{O})$, and for each vertex $v \in X$

(1) if $v \notin f^{-N}(\mathcal{O})$, then there exists a path $l_{v} \in \mathcal{P}$, unique up to direction, such that for every path $I \in \mathcal{I}_{N}$ which contains $v$ as an interior point, either $f^{N}(I) \notin \mathcal{P}$ or $f^{N}(I)=l_{v}^{ \pm 1} \in \mathcal{P}$ where \pm 1 denotes the direction, and

(2) if $v \in f^{-N}(\mathcal{O})$, then there exist paths $l_{i}, l_{t} \in \mathcal{P}$, unique up to direction, such that if $J_{1}$ and $J_{2}$ are elements of $\mathcal{I}_{N}$ such that $v$ is the terminal point of $J_{1}$ and the initial point of $J_{2}$, then $f^{N}\left(J_{1} J_{2}\right)=\left(l_{i} l_{t}\right)^{ \pm 1}$.

Fix $N$ satisfying the statement of Lemma 4.2. Let $\mathcal{I}=\left\{I \in \mathcal{I}_{N} \mid f^{N}(I) \in \mathcal{P}\right\}$. Then $I \in \mathcal{I}_{N} \backslash \mathcal{I}$ if and only if $f^{N}(I)$ is not locally one-to-one. Each $l \in \mathcal{P}$ can be represented as a product of $I_{i} \in \mathcal{I}_{N}$ such that the initial point of $I_{1}$ is the initial point of $l$, the terminal point of $I_{i}$ is the initial point of $I_{i+1}$ for $1 \leq i<j(l)$, and the terminal point of $I_{j(l)}$ is the terminal point of $l$ so that some $I_{i} \in \mathcal{I}$, some $I_{j} \in \mathcal{I}_{N} \backslash \mathcal{I}$, and $\tilde{f}^{N}(l)=f^{N}\left(I_{i(1)}\right) \cdots f^{N}\left(I_{i(l)}\right)$ where $I_{i(k)} \in \mathcal{I}$. Therefore each factor of $\tilde{f}^{N+i}(l)$ which is a path in $X$ is an element of $f^{i}(\mathcal{I})$ for every $l \in \mathcal{P}$ and $i \geq 0$.

Lemma 4.3. Suppose $l=f^{N}(I) \in \mathcal{P}$ for some $I \in \mathcal{I}$. Then every factor of $\tilde{f}^{i}(l)$ is an element of $f^{N}(\mathcal{I})$ for every nonnegative integer $i$.

Proof. Clearly every factor of $\tilde{f}^{(i)}(l)$ is an element of $f^{N}\left(\mathcal{I}_{N}\right)$. We must check that every factor is locally one-to-one. First consider the case that the image of $I$ is a subset of an edge of $X$. Assume that $I$ is represented as a product $J_{1} \cdots J_{j(I)}$ such that each $J_{j} \in \mathcal{I}_{N+i}$, and $f\left(J_{j}\right) \notin \mathcal{I}$ for some $j$. Then $f\left(J_{j}\right) \notin \mathcal{I}$ implies that 
$f^{N+i}\left(J_{j}\right)$ is not locally one-to-one on the image of $J_{j}$, and so $f^{N+1}(I)$ is not locally one-to-one. This contradicts the Nonfolding Axiom as we chose the image of $I$ to be a subset of an edge. So we have $f\left(J_{j}\right) \in \mathcal{I}$ for $1 \leq j \leq j(I)$.

Now suppose that $I$ contains a vertex $v$ of $X$ as an interior point. Let $I^{\prime} \in \mathcal{I}$ be a subset of an edge. Then there is a positive integer $k$ and a factor $J \in \mathcal{I}_{N+k}$ of $I^{\prime}$ such that $X \subset f^{k}\left(I^{\prime}\right)$ and $f^{k}(J)$ contains $v$ as an interior point. By Lemma 4.2 we have $f^{N}(I)=f^{N+k}(J)^{ \pm 1} \in \mathcal{P}$, and $\left.f^{N+i}\right|_{I}=\left.f^{N+k+i}\right|_{J}$ is locally one-to-one as $J$ is a subset of an edge. Therefore factors of $\tilde{f}^{i}\left(f^{N}(I)\right)$ are elements of $f^{N}(\mathcal{I})$ for all $i \geq 0$.

Definition 4.4 (Closed finite path set up to direction). The directed paths which are elements of $\mathcal{I}$ come in pairs, where one path in a pair is the other with reversed direction. Make a choice of one path from each pair and let $\mathcal{I}_{\text {or }}$ be the collection of chosen directed paths. Define

$$
\mathcal{P}_{\mathcal{O}}=\left\{f^{N}(I) \mid I \in \mathcal{I}_{\text {or }}\right\} .
$$

Then $\mathcal{P}_{\mathcal{O}}$ is a finite subset of $\mathcal{P}$ as $\mathcal{I}$ is a finite set.

Proposition 4.5. The set $\mathcal{P}_{\mathcal{O}}$ is the unique, up to the choice of direction, minimal subset of $\mathcal{P}$ satisfying the following conditions: $\tilde{f}(l) \in \mathcal{P}_{\mathcal{O}}^{*}$ for every $l \in \mathcal{P}_{\mathcal{O}}$, and $X \subset \bigcup_{l \in \mathcal{P}_{\mathcal{O}}} l$. There exists a positive integer $k$ such that $l_{1} \in \tilde{f}^{k}(l)$ for all $l_{1}, l \in \mathcal{P}_{\mathcal{O}}$.

Proof. By Lemma 4.3 $\tilde{f}(l)$ or $\tilde{f}(l)^{-1}$ is contained in $\mathcal{P}_{\mathcal{O}}^{*}$ for every $l \in \mathcal{P}_{\mathcal{O}}$. It remains to check minimality.

Suppose that $l=f^{N}(I)$ for some $I \in \mathcal{I}$. Then by Lemma 2.14, there exists a positive integer $j \geq N$ such that $X \subset f^{j}(I)$. If $l_{1}=f^{N}\left(I_{1}\right)$ such that $I_{1} \in \mathcal{I}$ and the interior of $I_{1}$ is contained in an edge $e_{1}$, then there exists a subpath $J_{1} \subset I$ such that $f^{j}\left(J_{1}\right)=I_{1}^{ \pm 1}$, and we have $f^{N}\left(I_{1}\right)=l_{1}^{ \pm 1}$ is a factor of $\tilde{f}^{j}\left(f^{N}(I)\right)$.

Next suppose that $l_{2}=f^{N}\left(I_{2}\right)$ where $I_{2}$ is a path in $\mathcal{I}$ such that $I_{2}$ contains a vertex $v$ as an interior point. Let $I_{3} \in \mathcal{I}$ be contained in an edge $e$, and $l_{3}=f^{N}\left(I_{3}\right)$. Then for some $m>0, v$ is the image under $f^{m}$ of an interior point of $e$, and by Lemma $4.2 l_{v}^{ \pm 1}$ is a factor of $\tilde{f}^{N+m}\left(l_{3}\right)$. This proves the minimality of $\mathcal{P}_{\mathcal{O}}$. and the uniqueness claim is also clear.

It is clear that $X \subset \bigcup_{l \in \mathcal{P}_{\mathcal{O}}} l$, and for all $l_{1}, l_{2} \in \mathcal{P}_{\mathcal{O}}$ there exists a positive integer $k=k(1,2)$ such that $l_{1}^{ \pm 1}$ is a factor of $\tilde{f}^{k}\left(l_{2}\right)$. Then the number $k$ can be chosen uniformly because for every $I \in \mathcal{I}$ such that the image of $I$ is contained in an edge, if $l=f^{N}(I)$, then $l^{ \pm 1}$ is a factor of $f^{m}\left(l^{\prime}\right)$ for every $l^{\prime} \in \mathcal{P}_{\mathcal{O}}$ and large $m$.

Definition 4.6 (Construction of new presentation). The new directed graph $X_{\mathcal{O}}$ defined by the set $\mathcal{O}$ has $n$ vertices and $m$ edges where $n$ is the cardinality of $\mathcal{O}$ and $m$ is the cardinality of $\mathcal{P}_{\mathcal{O}}$. The set of vertices $\mathcal{V}_{\mathcal{O}}$ of $X_{\mathcal{O}}$ corresponds to $\mathcal{O}$, and the set of edges $\mathcal{E}_{\mathcal{O}}$ corresponds to $\mathcal{P}_{\mathcal{O}}$ by the following rule: If $l_{i} \in \mathcal{P}_{\mathcal{O}}$ is a directed path from $v_{i, 1}$ to $v_{i, 2}$ in $X$ represented by a continuous map $\gamma_{l_{i}}:[0,1] \rightarrow X$ as in Notation 4.1, then $e_{i} \in \mathcal{E}_{\mathcal{O}}$ is a directed edge from $v_{i, 1}$ to $v_{i, 2}$ represented by a continuous map $\gamma_{i}:[0,1] \rightarrow e_{i}$ such that $\left.\gamma_{i}\right|_{(0,1)}$ is a homeomorphism, and $l_{i}^{-1}$ corresponds to $e_{i}^{-1}$ defined by $\gamma_{i}^{-1}:[0,1] \rightarrow e_{i}$ such that $\gamma_{i}^{-1}(t)=\gamma_{i}(1-t)$. So there is a natural projection $\rho_{\mathcal{O}}: X_{\mathcal{O}} \rightarrow X$ defined by $\gamma_{l_{i}} \circ\left(\gamma_{i}\right)^{-1}: e_{i} \rightarrow[0,1] \rightarrow l_{i}$, and the graph map $f_{\mathcal{O}}: X_{\mathcal{O}} \rightarrow X_{\mathcal{O}}$ is induced by $f: X \rightarrow X$ satisfying $\rho_{\mathcal{O}} \circ f_{\mathcal{O}}=f \circ \rho_{\mathcal{O}}$. Hence if $\tilde{f}_{\mathcal{P}}: l_{i} \mapsto l_{i, 1}^{s(1)} \cdots l_{i, m(i)}^{s(m(i))} \in \mathcal{P}_{\mathcal{O}}^{*}$ for $l_{i} \in \mathcal{P}_{\mathcal{O}}$, then $\tilde{f}_{\mathcal{O}}: e_{i} \mapsto e_{i, 1}^{s(1)} \cdots e_{i, m(i)}^{s(m(i))} \in \mathcal{E}_{\mathcal{O}}^{*}$ where $\rho_{\mathcal{O}}\left(e_{i}\right)=l_{i} \in \mathcal{E}_{\mathcal{O}}$ and $s(i)$ denotes the direction. 
Remark 4.7. Suppose that $\mathcal{P}_{\mathcal{O}}^{\prime}$ is $\mathcal{P}_{\mathcal{O}}$ with a different choice of directions of paths. Let $\left(X_{\mathcal{O}}, f_{\mathcal{O}}\right)$ be defined by $\mathcal{P}_{\mathcal{O}}$, and $\left(X_{\mathcal{O}}^{\prime}, f_{\mathcal{O}}^{\prime}\right)$ defined by $\mathcal{P}_{\mathcal{O}}^{\prime}$. Then the two graphs are the same except that the directions of some corresponding edges might be reversed, and it is easy to see that $f_{\mathcal{O}}$ and $f_{\mathcal{O}}^{\prime}$ are shift equivalent by graph maps.

Example 4.8. Let $X$ be a wedge of two circles $a, b$ with a unique vertex $p$, and $f: X \rightarrow X$ defined by $a \mapsto a a b$ and $b \mapsto a b$. So $(X, f)$ is given by the following diagram, in which $p$ is the vertex of $X$ and $\{q, r\}$ is a period 2 orbit.

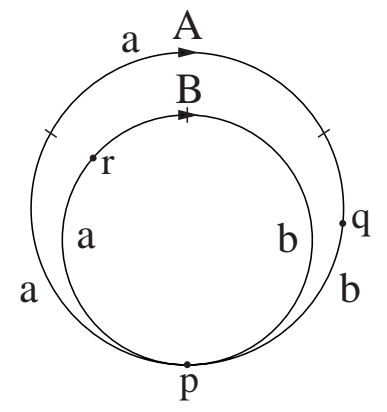

The set of directed paths $\mathcal{P}$ defined by the indicated periodic orbit $\{q, r\}$ is $\left\{\alpha, \beta, \gamma, \delta, \epsilon, \zeta, \alpha^{-1}, \beta^{-1}, \gamma^{-1}, \delta^{-1}, \epsilon^{-1}, \zeta^{-1}\right\}$ where $\alpha$ is the circle $A$ based at $q, \beta$ is the path from $q$ through $p$ to $r, \gamma$ is from $r$ through $p$ to $q, \delta$ is the circle $B$ based at $r, \epsilon$ is the path from $q$ through $p$ to $r$ with reverse direction to $B$, and $\zeta$ is the path from $r$ through $p$ with reverse direction to $B$ to $q$.

Every edge of $X$ has at least two points of $f^{-1}(\{q, r\})$, and it is not difficult to check that $f(\mathcal{I})=\left\{\alpha, \beta, \gamma, \alpha^{-1}, \beta^{-1}, \gamma^{-1}\right\}$. So $\mathcal{P}_{\{q, r\}}$ is $\{\alpha, \beta, \gamma\}$ up to the choice of direction, and the induced wrapping rule $\tilde{f}_{\mathcal{P}}: \mathcal{P} \rightarrow \mathcal{P}^{*}$ is given by

$$
\alpha \mapsto \gamma \alpha \beta, \quad \beta \mapsto \gamma, \quad \gamma \mapsto \beta \gamma \alpha \beta .
$$

Hence the new graph $X_{\{q, r\}}$ defined by $\{q, r\}$ is the following graph.

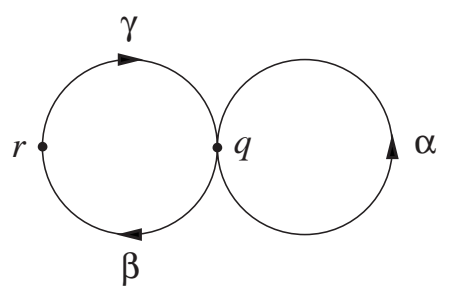

Remark 4.9. To compute the graph $X_{\mathcal{O}}$ and the map $f_{\mathcal{O}}$, we don't need to find the integer $N$ and $\mathcal{I}$ given in Lemma 4.2 If we choose a path $l \in \mathcal{P}$, and iteratively apply $\tilde{f}$ to the factors of $\tilde{f}^{n}(l)$ which are paths, we will eventually generate a set of paths invariant under $\tilde{f}$, and this set will contain $\mathcal{P}_{\mathcal{O}}$.

Example 4.10. Suppose that $(Y, g)$ is given by Figure 3 and $p$ is a fixed point of $g$.

Then the set $\mathcal{P}$ of directed paths based at $p$ is an infinite set for if we call $a_{1}$ the path from $p$ to the branch point in the edge $a$ and $a_{2}$ the path from the branch point to $p$, then the paths $a_{1} b \underbrace{d \cdots d}_{n} c a_{2}$ are in $\mathcal{P}$. 


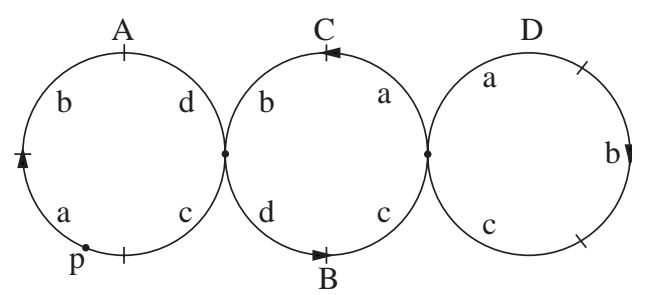

FiguRe 3. $(Y, g)$ with a fixed point $p$

If we choose $\ell$ as the loop $a_{1} a_{2}$ based at $p$, then $\tilde{g}(\ell)=a_{1} b d c a_{2}$ and $\tilde{g}^{2}(\ell)=$ $a_{1} b d d c a_{2} a_{1} b c a_{2} a_{1} b c a_{2} a_{1} b c a_{2}$.

For $e=a_{1} b d d c a_{2}$ and $f=a_{1} b c a_{2}, \tilde{g}: e \mapsto e f f f$ and $f \mapsto e f$. So $\{e, f\}$ is a closed minimal subset of $\mathcal{P}$, and $\mathcal{P}_{\{p\}}=\{e, f\}$ by uniqueness. The new graph $\left(Y_{\{p\}}, g_{\{p\}}\right)$ defined by $\{p\}$ is the following graph.

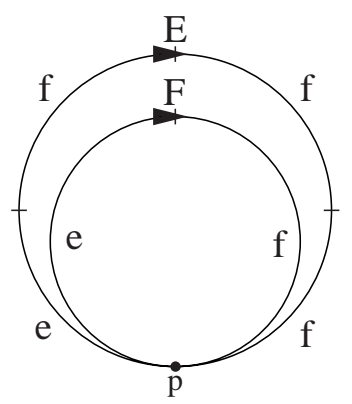

Example 4.11. Suppose that $Z$ is given in the following graph, that $h: Z \rightarrow Z$ is given by $a \mapsto b a b^{-1}$ and $b \mapsto a b a^{-1}$, and that $p$ is a fixed point.

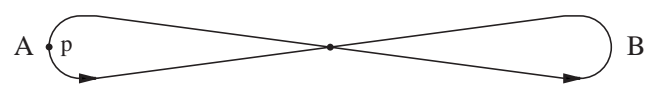

Let $a_{1}$ denote the path from $p$ to the branch point, $a_{2}$ the path from the branch point to $p$, and $\ell=a_{2} b a_{1}$. Then $\tilde{h}(\ell)=a_{2} b^{-1} a_{1} a_{2} b a_{2}^{-1} a_{1}^{-1} b a_{1}$ and for $\alpha=a_{2} b^{-1} a_{1}$, $\beta=a_{2} b a_{2}^{-1}$ and $\gamma=a_{1}^{-1} b a_{1}$,

$$
\tilde{h}: \alpha \mapsto \alpha \beta^{-1} \gamma, \quad \beta \mapsto \alpha \beta \alpha^{-1}, \quad \gamma \mapsto \gamma^{-1} \beta \gamma .
$$

The new graph $Z_{\{p\}}$ defined by $\{p\}$ is a wedge of three circles as in the graph below.

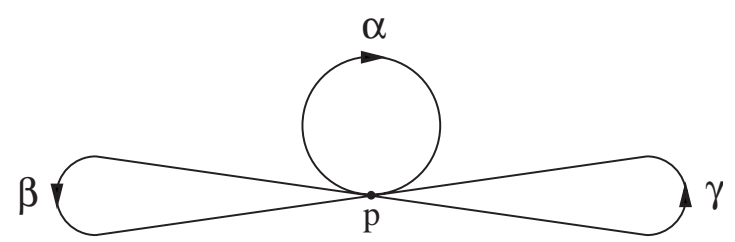


Theorem 4.12. Suppose that $(X, f)$ satisfies all six axioms, that $\mathcal{O}$ is a finite subset of $X$ such that $f(\mathcal{O})=\mathcal{O}$, and that $\mathcal{P}_{\mathcal{O}}$ and $\left(X_{\mathcal{O}}, f_{\mathcal{O}}\right)$ are the minimal closed subsets of $\mathcal{P}$ and the new presentation defined by $\mathcal{O}$, respectively. Then the natural projection $\rho_{\mathcal{O}}: X_{\mathcal{O}} \rightarrow X$ gives a conjugacy from $\left(\bar{X}_{\mathcal{O}}, \bar{f}_{\mathcal{O}}\right)$ to $(\bar{X}, \bar{f})$.

Proof. We will show that $\rho=\rho_{\mathcal{O}}$ is part of a shift equivalence, that is, we will define a continuous map $\psi: X \rightarrow X_{\mathcal{O}}$ and a positive integer $m$ such that

$$
f_{\mathcal{O}} \circ \psi=\psi \circ f, \rho \circ \psi=f^{m} \text {, and } \psi \circ \rho=f_{\mathcal{O}}^{m} .
$$

Recall $\mathcal{P}_{\mathcal{O}}=\left\{f^{N}(I) \mid I \in \mathcal{I}_{\text {or }}\right\}$ for some fixed choice of a subset $\mathcal{I}_{\text {or }}$ of $\mathcal{I}_{N}$ as in Definition 4.4. By the choice of $N$ (from Lemma 4.2), every $I \in \mathcal{I}_{\text {or }}$ contains at most one vertex of $X$. It is trivial that if $x \in X \backslash f^{-N}(\mathcal{O})$ is contained in some $I \in \mathcal{I}_{\text {or }}$ and the interior of $I$ does not contain any vertex of $X$, then $I$ is the unique element in $\mathcal{I}_{\text {or }}$ which contains $x$.

If $v$ is a vertex of $X$, then by Lemma 4.2, $v \in f^{-N}(\mathcal{O})$ or there is a neighborhood $U_{v}$ of $v$ such that $U_{v}$ is the union of all paths in $\mathcal{I}$ containing $v$ as an interior point and $f^{N}\left(U_{v}\right)$ is the image of $l_{v} \in \mathcal{P}_{\mathcal{O}}$. Hence, for every $x \in X \backslash f^{-N}(\mathcal{O})$, there is a unique path $l_{x} \in \mathcal{P}_{\mathcal{O}}$ such that if $x \in I \in \mathcal{I}_{\text {or }}$, then $f^{N}(I)=l_{x}^{ \pm 1}$.

For $x \in X \backslash f^{-N}(\mathcal{O})$, let $e_{x}$ be the edge of $X_{\mathcal{O}}$ corresponding to $l_{x}$. We will define $\psi(x)$ to be the appropriate point $x_{\mathcal{O}}$ in $e_{x}$ satisfying $\rho \circ \psi(x)=f^{N}(x)$. Let $\gamma:[0,1] \rightarrow e_{x}$ be the continuous function (in the equivalence class $l_{x}$ ) associated to $e_{x}$ in the definition of $X_{\mathcal{O}}$ and $\rho$. Fix $I$ in $\mathcal{I}_{\text {or }}$ such that $x \in I$. For the moment let $I$ also denote a specific map $[0,1] \rightarrow X$. Then there is a homeomorphism $h:[0,1] \rightarrow[0,1]$ such that $\rho \circ \gamma=f^{N} \circ I \circ h$. Let $t$ be the unique number in $(0,1)$ such that $I \circ h(t)=x$, and define $x_{\mathcal{O}}=\gamma(t)$. Then $\rho \circ \psi(x)=\rho\left(x_{\mathcal{O}}\right)=\rho \circ \gamma(t)=$ $f^{N} \circ I \circ h(t)=f^{N}(x)$ as required.

For $x \in f^{-N}(\mathcal{O})$, we define $\psi(x)$ as the unique point in $X_{\mathcal{O}}$ which $\rho$ maps to $f^{N}(x) \in \mathcal{O}$. Then $\psi$ is continuous, $\rho \circ \psi=f^{N}$, and clearly $f \circ \rho=\rho \circ f_{\mathcal{O}}$ by Definition 4.6. Consequently $\rho \circ \psi \circ \rho=f^{N} \circ \rho=\rho \circ f_{\mathcal{O}}^{N}$. Therefore the two maps $\psi \circ \rho$ and $f_{\mathcal{O}}^{N}$ send any given edge (considered as a path) in $X_{\mathcal{O}}$ to paths which $\rho$ sends to the same concatenation of elements of $\mathcal{I}$. Such a concatenation has a unique lifting under $\rho$, therefore $\psi \circ \rho=f_{\mathcal{O}}^{N}$.

It remains to show that $f_{\mathcal{O}} \circ \psi=\psi \circ f$. Because $\rho$ is surjective, it suffices to show $f_{\mathcal{O}} \circ \psi \circ \rho=\psi \circ f \circ \rho$, and this is true because

$$
f_{\mathcal{O}} \circ \psi \circ \rho=f_{\mathcal{O}}^{N+1}=(\psi \circ \rho) \circ f_{\mathcal{O}}=\psi \circ\left(\rho \circ f_{\mathcal{O}}\right)=\psi \circ(f \circ \rho) .
$$

Therefore $\left(X_{\mathcal{O}}, f_{\mathcal{O}}\right)$ is shift equivalent to $(X, f)$, and $\bar{\rho}_{\mathcal{O}}$ is a topologically conjugacy by Proposition 3.7

Remarks 4.13. (1) Theorem 4.12 requires the Flattening Axiom. See Example 4.17.

(2) If $\mathcal{O}$ is not a subset of $\mathcal{V}_{X}$, then the shift equivalence maps $\rho$ and $\psi$ in Theorem 4.12 cannot be graph maps.

Remark 4.14 (Preperiodic sets). If $\mathcal{F}$ is a finite subset of $X$ such that $f(\mathcal{F}) \subseteq \mathcal{F}$, then it is not difficult to apply the graph algorithm to $\mathcal{F}$ so that we have a finite graph with a graph map $\left(X_{\mathcal{F}}, f_{\mathcal{F}}\right)$. And there is a positive integer $k=k_{\mathcal{F}}$ such that $f^{k}(\mathcal{F})=f^{k+i}(\mathcal{F})$ for all $i \geq 0$. Let $\mathcal{O}_{\mathcal{F}}=f^{k}(\mathcal{F})$.

We get $f$ and $f_{\mathcal{F}}$ shift equivalent just as before. Now it is not hard to check that $f_{\mathcal{F}}$ and $f_{\mathcal{O}_{\mathcal{F}}}$ are shift equivalent by graph maps. Hence the associated SFT 
covers of the next section will be conjugate for $\mathcal{F}$ and $\mathcal{O}_{\mathcal{F}}$, and that is why we only concern ourselves with sets $\mathcal{O}$ which are unions of periodic orbits.

Elementary presentation. One interesting application of the graph algorithm is the elementary presentations of solenoids. In [15, §5], Williams introduced an elementary presentation of a solenoid in which $X$ is a wedge of circles and $f$ leaves the unique branch point of $X$ fixed. And he showed in [15] Theorem 5.2] that, for every generalized one-dimensional solenoid with shift map $(\bar{X}, \bar{f})$, there exists an integer $m$ such that $\left(\bar{X}, \overline{f^{m}}\right)$ has an elementary presentation. We can improve his theorem by getting sharp bounds on $m$.

Suppose that $(X, f)$ satisfies all six axioms, and that $a$ is a fixed point of $f^{m}$. For $\left(X, f^{m}\right)$, let $\left(X_{\{a\}}, f_{\{a\}}^{m}\right)$ be the new presentation defined by $\{a\}$. Then the new graph $X_{\{a\}}$ has only one vertex $a$ which is a fixed point by $f_{\{a\}}^{m}: X_{\{a\}} \rightarrow X_{\{a\}}$, and each edge in $X_{\{a\}}$ is homeomorphic to a circle. So $\left(X_{\{a\}}, f_{\{a\}}^{m}\right)$ is an elementary presentation and $\left(\bar{X}, \bar{f}^{m}\right)$ is conjugate to $\left(\bar{X}_{\{a\}}, \bar{f}_{\{a\}}\right)$ by Theorem 4.12 . More generally, we have the following proposition:

Proposition 4.15. For a given 1-solenoid, the minimal number of vertices in a presentation $(X, f)$ is the minimal period of points in $X$. In particular, $(\bar{X}, \bar{f})$ has an elementary presentation if and only if $f: X \rightarrow X$ has a fixed point.

Remark 4.16. Williams showed that two elementary presented solenoid systems $\left(\bar{Y}_{1}, \bar{g}_{1}\right)$ and $\left(\bar{Y}_{2}, \bar{g}_{2}\right)$ are topologically conjugate to each other if and only if the shift equivalence classes of $g_{1 *}: \pi_{1}\left(Y_{1}, y_{1}\right) \rightarrow \pi_{1}\left(Y_{1}, y_{1}\right)$ and $g_{2 *}: \pi_{1}\left(Y_{2}, y_{2}\right) \rightarrow \pi_{1}\left(Y_{2}, y_{2}\right)$ are the same where $y_{i}$ is the unique branch point of $Y_{i}$ for $i=1,2$ ([15] 7.3]). Proposition 4.15 extends the range of Williams' classification theorem.

Example 4.17. Theorem 4.12 and Proposition 4.15 require the Flattening Axiom.

Let $X$ be a wedge of two circles $a$ and $b$ with $f: X \rightarrow X$ defined by

$$
a \mapsto b b a, \quad b \mapsto a b b .
$$

Then $(X, f)$ is an elementary presentation of a branched solenoid and $f$ does not satisfy the Flattening Axiom. The circle $b$ contains a unique nonbranch fixed point $q$.

The directed path set $\mathcal{P}_{\{q\}}$ has three loops

$$
\alpha=b_{2} b_{1}, \quad \beta=b_{2} a b_{2}, \quad \gamma=b_{2} a a b_{1}
$$

where $b_{1}$ is the arc from the branch point to $q$ and $b_{2}$ is from $q$ to the branch point.

Let $X_{\{q\}}$ be a wedge of three circles $\alpha, \beta, \gamma$ based at $q$, and $f_{\{q\}}: X_{\{q\}} \rightarrow X_{\{q\}}$ the map induced from $f: X \rightarrow X$ by

$$
\alpha \mapsto \alpha \beta, \quad \beta \mapsto \alpha \alpha \alpha \gamma, \quad \gamma \mapsto \alpha \alpha \alpha \beta \alpha \gamma
$$

Then $\left(X_{\{q\}}, f_{\{q\}}\right)$ satisfies the Flattening Axiom. So $(\bar{X}, \bar{f})$ is not topologically conjugate to $\left(\bar{X}_{\{q\}}, \bar{f}_{\{q\}}\right)$.

\section{CAnOnical SFT COVERS}

In $\S 4$, we constructed a new presentation $\left(X_{\mathcal{O}}, f_{\mathcal{O}}\right)$ for the given presentation $(X, f)$ and the finite invariant set $\mathcal{O}$ of $X$. Associated to this new presentation, we have an $\operatorname{SFT}\left(\Sigma_{\mathcal{O}}, \sigma_{\mathcal{O}}\right)$ defined by $\left(\mathcal{E}_{X_{\mathcal{O}}}, \tilde{f}_{\mathcal{O}}\right)$ and a semiconjugacy $p_{\mathcal{O}}:\left(\Sigma_{\mathcal{O}}, \sigma_{\mathcal{O}}\right) \rightarrow$ $\left(X_{\mathcal{O}}, f_{\mathcal{O}}\right)$ constructed by the standard algorithm described in Section 2. We will show that this new SFT cover defined by a finite invariant set is canonical. As an 
application, we show that two solenoid systems are not conjugate by comparing Bowen-Franks groups of their SFT covers defined by periodic orbits of the same period.

Suppose that $(X, f)$ satisfies all six axioms, that $\mathcal{O}$ is a finite subset of $X$ such that $f(\mathcal{O})=\mathcal{O}$, and that $\left(X_{\mathcal{O}}, f_{\mathcal{O}}\right)$ is the presentation defined by $(X, f)$ and $\mathcal{O}$. If $\mathcal{E}_{\mathcal{O}}$ is the set of edges in $X_{\mathcal{O}}$, and $\tilde{f}_{\mathcal{O}}: \mathcal{E}_{\mathcal{O}} \rightarrow \mathcal{E}_{\mathcal{O}}^{*}$ is the wrapping rule associated to $f_{\mathcal{O}}$, then $\left(\mathcal{E}_{\mathcal{O}}, \tilde{f}_{\mathcal{O}}\right)$ defines a two-sided $\operatorname{SFT}\left(\Sigma_{\mathcal{O}}, \sigma_{\mathcal{O}}\right)$.

There is a well-defined quotient map $p_{\mathcal{O}}: \Sigma_{\mathcal{O}} \rightarrow \bar{X}_{\mathcal{O}}$ such that $\bar{f}_{\mathcal{O}} \circ p_{\mathcal{O}}=p_{\mathcal{O}} \circ \sigma_{\mathcal{O}}$. If $\rho_{\mathcal{O}}: X_{\mathcal{O}} \rightarrow X$ is the natural projection which maps each edge $e$ of $X_{\mathcal{O}}$ to the corresponding path $l$ in $X$, then $\bar{\rho}_{\mathcal{O}}: \bar{X}_{\mathcal{O}} \rightarrow \bar{X}$ is a conjugacy by Theorem 4.12 and $\bar{\rho}_{\mathcal{O}} \circ p_{\mathcal{O}}: \Sigma_{\mathcal{O}} \rightarrow \bar{X}$ is a finite-to-one quotient map.

The canonical projection map to the zeroth coordinate $\pi: \bar{X} \rightarrow X$ induces a bijection $\overline{\mathcal{O}} \leftrightarrow \mathcal{O}$ of finite invariant sets of $\bar{X}$ and $X$. We will call $\left(\Sigma_{\mathcal{O}}, \sigma_{\mathcal{O}}\right)$ the SFT cover of $(\bar{X}, \bar{f})$ defined by $\mathcal{O}$ or by $\overline{\mathcal{O}}$.

Example 5.1. Let $(X, f)$ be as in Example 4.8 $p$ the branch point of $X$ which is a fixed point of $f$, and $\{q, r\}$ a period 2 orbit. Then the 'natural' SFT cover of $(\bar{X}, \bar{f})$ is the SFT $\left(\Sigma_{\{p\}}, \sigma_{\{p\}}\right)$ defined by the orbit $\{p\}$. From the wrapping rule $\tilde{f}: a \mapsto a a b, b \mapsto a b$ we see that $\left(\Sigma_{\{p\}}, \sigma_{\{p\}}\right)$ is represented by the following adjacency matrix:

$$
M_{\{p\}}=\left(\begin{array}{ll}
2 & 1 \\
1 & 1
\end{array}\right) .
$$

The induced map $\tilde{f}_{\{q, r\}}: \mathcal{P}_{\{q, r\}} \rightarrow \mathcal{P}_{\{q, r\}}^{*}$ is given by

$$
\alpha \mapsto \gamma \alpha \beta, \quad \beta \mapsto \gamma, \quad \gamma \mapsto \beta \gamma \alpha \beta .
$$

So the SFT cover $\left(\Sigma_{\{q, r\}}, \sigma_{\{q, r\}}\right)$ of $(\bar{X}, \bar{f})$ defined by $\{q, r\}$ is given by the following matrix:

$$
M_{\{q, r\}}=\left(\begin{array}{ccc}
1 & 1 & 1 \\
0 & 0 & 1 \\
1 & 2 & 1
\end{array}\right) .
$$

Remark that $\left(\bar{X}_{\{p\}}, \bar{f}_{\{p\}}\right)$ is topologically conjugate to $\left(\bar{X}_{\{q, r\}}, \bar{f}_{\{q, r\}}\right)$ by Theorem 4.12. But $\left(\Sigma_{\{p\}}, \sigma_{\{p\}}\right)$ is not topologically conjugate to $\left(\Sigma_{\{q, r\}}, \sigma_{\{q, r\}}\right)$ as the trace of $M_{\{p\}}$ is different from that of $M_{\{q, r\}}$.

Theorem 5.2. Suppose that $(X, f)$ and $(Y, g)$ satisfy all six Axioms, and that $(\bar{X}, \bar{f})$ is topologically conjugate to $(\bar{Y}, \bar{g})$ by a conjugacy map $\phi$. If $\overline{\mathcal{O}}$ is a finite union of periodic orbits of $\bar{f}$ and $\overline{\mathcal{O}}^{\prime}=\phi(\overline{\mathcal{O}})$, then there is a unique conjugacy $\tilde{\phi}:\left(\Sigma_{\mathcal{O}}, \sigma_{\mathcal{O}}\right) \rightarrow\left(\Sigma_{\mathcal{O}^{\prime}}, \sigma_{\mathcal{O}^{\prime}}\right)$ such that the following diagram commutes:

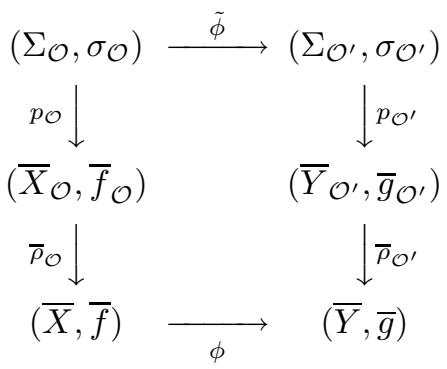


Proof. That $\phi$ is a conjugacy implies that $\overline{\mathcal{O}}^{\prime}$ is a finite union of periodic orbits of $\bar{g}$. Let $\left(X_{\mathcal{O}}, f_{\mathcal{O}}\right)$ and $\left(Y_{\mathcal{O}^{\prime}}, g_{\mathcal{O}^{\prime}}\right)$ be the new graphs with graph maps defined by $\mathcal{O}=\pi(\overline{\mathcal{O}})$ and $\mathcal{O}^{\prime}=\pi\left(\overline{\mathcal{O}}^{\prime}\right)$, respectively. Then by Theorem 4.12, there exist shift equivalence maps $\left(\rho_{\mathcal{O}}, \psi_{\mathcal{O}}\right)$ for $\left(X_{\mathcal{O}}, f_{\mathcal{O}}\right)$ and $(X, f)$, and $\left(\rho_{\mathcal{O}^{\prime}}, \psi_{\mathcal{O}^{\prime}}\right)$ for $\left(Y_{\mathcal{O}^{\prime}}, g_{\mathcal{O}^{\prime}}\right)$ and $(Y, g)$. Let $\phi_{\mathcal{O}}: \bar{X}_{\mathcal{O}} \rightarrow \bar{Y}_{\mathcal{O}^{\prime}}$ be the conjugacy $\left(\bar{\rho}_{\mathcal{O}^{\prime}}\right)^{-1} \circ \phi \circ \bar{\rho}_{\mathcal{O}}$, which lifts $\phi$.

By the Ladder Lemma, there is a shift equivalence $r_{\mathcal{O}}: X_{\mathcal{O}} \rightarrow Y_{\mathcal{O}^{\prime}}$ and $s_{\mathcal{O}}: Y_{\mathcal{O}^{\prime}} \rightarrow$ $X_{\mathcal{O}}$ such that $\bar{r}_{\mathcal{O}}=\phi_{\mathcal{O}}$ and $\bar{s}_{\mathcal{O}}=\phi_{\mathcal{O}}^{-1}$. Since $\phi$ sends $\overline{\mathcal{O}}$ to $\overline{\mathcal{O}}^{\prime}, r_{\mathcal{O}}$ and $s_{\mathcal{O}}$ are graph maps. Then it follows from Theorem 3.8 that there is a unique conjugacy $\tilde{\phi}:\left(\Sigma_{\mathcal{O}}, \sigma_{\mathcal{O}}\right) \rightarrow\left(\Sigma_{\mathcal{O}^{\prime}}, \sigma_{\mathcal{O}^{\prime}}\right)$ lifting $\phi_{\mathcal{O}}$. Therefore $\tilde{\phi}$ is the unique conjugacy lifting $\phi$.

Remarks 5.3. (1) It is necessary to assume $\overline{\mathcal{O}}^{\prime}=\phi(\overline{\mathcal{O}})$. See Example 5.1

(2) We need the Flattening Axiom to guarantee that $\phi_{\mathcal{O}}:\left(\bar{X}_{\mathcal{O}}, \bar{f}_{\mathcal{O}}\right) \rightarrow\left(\bar{Y}_{\mathcal{O}^{\prime}}, \bar{g}_{\mathcal{O}^{\prime}}\right)$ is a conjugacy. See Remark4.13 and Example 4.17

Bowen-Franks groups. We say that two dynamical systems $(M, \phi)$ and $\left(M^{\prime}, \phi^{\prime}\right)$ are flow equivalent if they have topologically equivalent suspension flows.

Definition $5.4([9, \S 7])$. Let $A$ be an $r \times r$ integral matrix. The Bowen-Franks group of $A$ is

$$
B F(A)=\operatorname{coker}(I d-A)=\mathbb{Z}^{r} / \mathbb{Z}^{r}(I d-A),
$$

where $\mathbb{Z}^{r}(I d-A)$ is the image of $\mathbb{Z}^{r}$ under the matrix $I d-A$ acting on the right.

If two irreducible SFTs are flow equivalent, then they have isomorphic BowenFranks group ([5]).

Example 5.5. Williams posed the following question ([13, 15]): If $X$ is a wedge of two circles $a, b$, and $g_{1}, g_{2}: X \rightarrow X$ are given by

$$
\begin{aligned}
& \tilde{g}_{1}(a)=a a b b a, \quad \tilde{g}_{1}(b)=a, \text { and } \\
& \tilde{g}_{2}(a)=a b a b a, \quad \tilde{g}_{2}(b)=a,
\end{aligned}
$$

then are $g_{1}$ and $g_{2}$ shift equivalent?

Ustinov ([13]) already showed that they are not shift equivalent to each other using ideas of combinatorial group theory. We will give an alternate argument using the canonical SFT covers. We will compare the Bowen-Franks groups of the SFT covers defined by period- 2 orbits. Since conjugacy preserves flow equivalence classes, it suffices to show that there is no bijection between the SFT covers defined by period 2 orbits in $\left(X, g_{1}\right)$ and $\left(X, g_{2}\right)$, respectively, such that the bijection respects the Bowen-Franks groups.

The presentations $\left(X, g_{1}\right)$ and $\left(X, g_{2}\right)$ are given by the following diagrams. So $A$ has three subpaths $a_{1}, a_{2}, a_{3}$ which map to $A$ by $g_{i}$, and two subpaths $b_{1}, b_{2}$ which map to $B$.
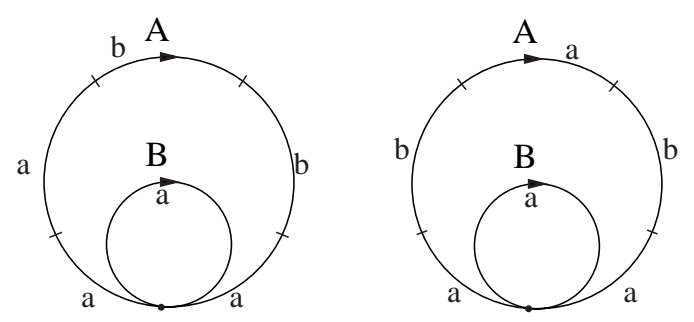
Each presentation has five period 2 orbits (excluding fixed points). Let us denote $\left(a_{i}, a_{j}\right)$ as the period 2 orbit contained in $a_{i} \cup a_{j}$ and $\left(b_{k}, B\right)$ as the period 2 orbit in $b_{k} \cup B$. For $\left(X, g_{1}\right)$, the SFT covers defined by period 2 orbits are represented by the following matrices:

$$
\begin{gathered}
M\left(a_{1}, a_{2}\right)=\left(\begin{array}{lll}
0 & 1 & 0 \\
3 & 1 & 1 \\
5 & 3 & 1
\end{array}\right), M\left(a_{1}, a_{3}\right)=\left(\begin{array}{lll}
1 & 2 & 0 \\
1 & 1 & 1 \\
3 & 4 & 0
\end{array}\right), M\left(a_{2}, a_{3}\right)=\left(\begin{array}{lll}
2 & 3 & 0 \\
0 & 0 & 1 \\
4 & 5 & 0
\end{array}\right), \\
M\left(b_{1}, B\right)=\left(\begin{array}{llll}
2 & 1 & 1 & 1 \\
1 & 0 & 1 & 1 \\
1 & 0 & 0 & 0 \\
2 & 1 & 0 & 0
\end{array}\right), \text { and } M\left(b_{2}, B\right)=\left(\begin{array}{llll}
2 & 1 & 1 & 1 \\
1 & 0 & 0 & 1 \\
1 & 0 & 0 & 0 \\
2 & 1 & 1 & 0
\end{array}\right)
\end{gathered}
$$

and for $\left(X, g_{2}\right)$,

$$
\begin{gathered}
N\left(a_{1}, a_{2}\right)=\left(\begin{array}{lll}
0 & 0 & 1 \\
3 & 1 & 1 \\
4 & 2 & 1
\end{array}\right), N\left(a_{1}, a_{3}\right)=\left(\begin{array}{lll}
1 & 0 & 2 \\
2 & 1 & 0 \\
3 & 2 & 0
\end{array}\right), N\left(a_{2}, a_{3}\right)=\left(\begin{array}{lll}
1 & 3 & 1 \\
0 & 0 & 1 \\
2 & 4 & 1
\end{array}\right) \\
N\left(b_{1}, B\right)=\left(\begin{array}{lll}
1 & 2 & 2 \\
1 & 1 & 2 \\
1 & 1 & 0
\end{array}\right), \text { and } N\left(b_{2}, B\right)=\left(\begin{array}{lll}
1 & 2 & 2 \\
1 & 0 & 1 \\
1 & 2 & 1
\end{array}\right)
\end{gathered}
$$

We indicate the computation of $M\left(a_{1}, a_{2}\right)$ as an example. The points in the periodic orbit $\left(a_{1}, a_{2}\right)$ and the new graph $X_{\left(a_{1}, a_{2}\right)}$ are given in the following diagrams.
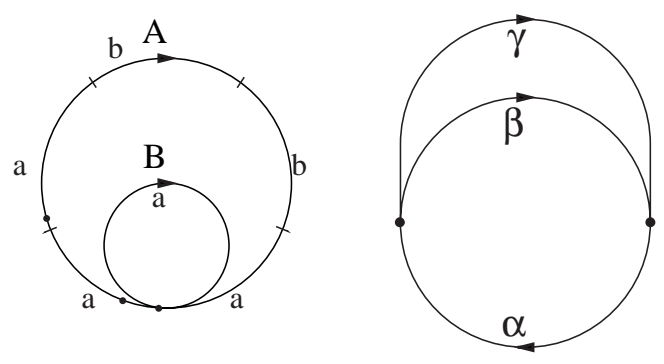

Let $p_{1}$ denote the path from the branch point to the point in $a_{1}, p_{2}$ the path from the point in $a_{1}$ to the point in $a_{2}$, and $p_{3}$ the path from the point in $a_{2}$ to the branch point. Let $\alpha=p_{2}, \beta=p_{3} p_{1}$, and $\gamma=p_{3} b b p_{1}$. Then the substitution rule $\tilde{g}_{\left(a_{1}, a_{2}\right)}: \mathcal{E}_{\left(a_{1}, a_{2}\right)} \rightarrow \mathcal{E}_{\left(a_{1}, a_{2}\right)}^{*}$ is given by

$$
\alpha \mapsto \beta, \quad \beta \mapsto \alpha \gamma \alpha \beta \alpha, \quad \gamma \mapsto \alpha \gamma \alpha \beta \alpha \beta \alpha \beta \alpha .
$$

Use the Smith form to compute Bowen-Franks groups (9, §7.4]) of SFT covers defined by period 2 orbits. Then it is not difficult to obtain that in $\left(X, g_{1}\right), M\left(a_{1}, a_{3}\right)$ and $M\left(b_{1}, B\right)$ have $\mathbb{Z}_{2} \oplus \mathbb{Z}_{4}$, and $M\left(a_{1}, a_{2}\right), M\left(a_{2}, a_{3}\right)$ and $M\left(b_{2}, B\right)$ have $\mathbb{Z}_{8}$ as their Bowen-Franks groups. And in $\left(X, g_{2}\right), N\left(a_{1}, a_{2}\right), N\left(a_{1}, a_{3}\right)$ and $N\left(a_{2}, a_{3}\right)$ have $\mathbb{Z}_{2} \oplus \mathbb{Z}_{4}$, and $N\left(b_{1}, B\right), N\left(b_{2}, B\right)$ have $\mathbb{Z}_{8}$. So the number of SFT covers which have the same Bowen-Franks groups are different, and $\bar{g}_{1}$ is not conjugate to $\bar{g}_{2}$. Therefore $\left(X, g_{1}\right)$ is not shift equivalent to $\left(X, g_{2}\right)$. 


\section{Appendix A. ONE-SIDED SFT}

Suppose that $(X, f)$ and $(Y, g)$ are presentations of solenoids which are shift equivalent to each other by a shift equivalence $r: X \rightarrow Y$ and $s: Y \rightarrow X$. Assume that $\mathcal{O}$ is a finite invariant subset of $(X, f)$ and $\mathcal{O}^{\prime}=r(\mathcal{O})$, and denote $\left(\Sigma_{\mathcal{O}}, \sigma_{\mathcal{O}}\right)$ and $\left(\Sigma_{\mathcal{O}^{\prime}}, \sigma_{\mathcal{O}^{\prime}}\right)$ as the SFT covers of $(\bar{X}, \bar{f})$ and $(\bar{Y}, \bar{g})$ defined by $\mathcal{O}$ and $\mathcal{O}^{\prime}$ respectively. Then $\left(\Sigma_{\mathcal{O}}, \sigma_{\mathcal{O}}\right)$ and $\left(\Sigma_{\mathcal{O}^{\prime}}, \sigma_{\mathcal{O}^{\prime}}\right)$ are defined by nonnegative integer matrices $M_{\mathcal{O}}$ and $M_{\mathcal{O}^{\prime}}$, respectively. And we can make one-sided SFTs $\left(S_{\mathcal{O}}, \sigma_{\mathcal{O}}\right)$ and $\left(S_{\mathcal{O}^{\prime}}, \sigma_{\mathcal{O}^{\prime}}\right)$ from $M_{\mathcal{O}}$ and $M_{\mathcal{O}^{\prime}}$, respectively.

The purpose of this appendix is to give an example in which the one-sided SFTs $\left(S_{\mathcal{O}}, \sigma_{\mathcal{O}}\right)$ and $\left(S_{\mathcal{O}^{\prime}}, \sigma_{\mathcal{O}^{\prime}}\right)$ are not conjugate.

Example A.1. Let $\left(Y_{\{p\}}, g_{\{p\}}\right)$ be as in Example 4.10 Let $Y_{1}$ be a graph such that $\mathcal{E}_{Y_{1}}=\{1,2,3,4,5,6,7,8\}$ with a graph map $g_{1}: Y_{1} \rightarrow Y_{1}$ defined by

$$
1 \mapsto 1234357, \quad 2,4,5,6 \mapsto 8,3 \mapsto 167, \quad 7 \mapsto 1 \text {, and } 8 \mapsto 678
$$

The presentation $\left(Y_{\{p\}}, g_{\{p\}}\right)$ and the graph $Y_{1}$ are given in the following diagrams. In $Y_{1}$, the fixed point labeled $p$ is the terminal point of the edge 8 and the initial point of the edge 1 .
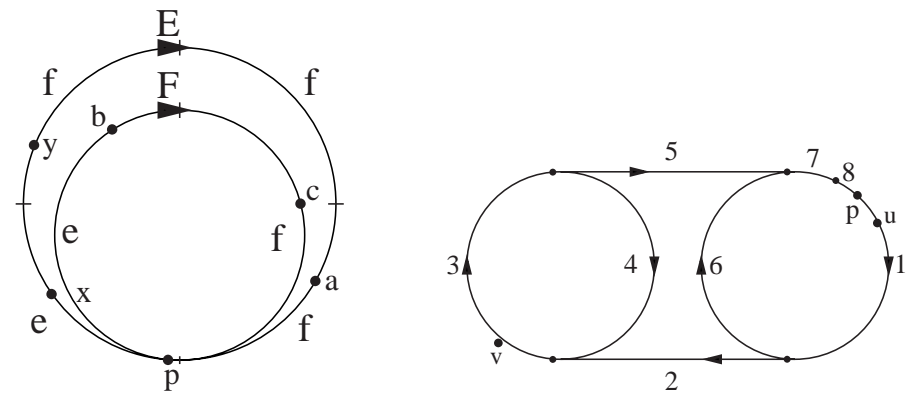

We will define a lag 1 shift equivalence of $g_{\{p\}}$ and $g_{1}$ by graph maps $r, s$ under which the points labeled $p$ correspond. The points labeled $x, y$ in $Y_{\{p\}}$ and $u, v$ in $Y_{1}$ form period two orbits of $g_{\{p\}}$ and $g_{1}$, respectively. The map $r$ will send the points $x, y$ to the points $u, v$. But, we will see that the covering SFTs associated to $\{x, y\}$ and $\{u, v\}$ are not conjugate as one-sided shifts.

The points $a, b$ in $Y_{\{p\}}$ is a period two orbit, and the point $c$ is the unique point in the edge $F$ such that $g_{\{p\}}(c)=b$. Let $e_{1}$ be the path from $p$ to $a, e_{2}$ the path from $a$ to $p, f_{1}$ the path from $p$ to $b, f_{2}$ the path from $b$ to $c$, and $f_{3}$ the path from $c$ to $p$. Define $r: Y_{\{p\}} \rightarrow Y_{1}$ and $s: Y_{1} \rightarrow Y_{\{p\}}$ by

$$
\begin{aligned}
& \tilde{r}: e_{1} \mapsto 1234357, e_{2} \mapsto 8, f_{1} \mapsto 1, f_{2} \mapsto 67, f_{3} \mapsto 8, \\
& \tilde{s}: 1 \mapsto e_{1}, 2,6 \mapsto e_{2}, 3 \mapsto f_{1} f_{2}, 4,5 \mapsto f_{3}, 7 \mapsto f_{1}, 8 \mapsto f_{2} f_{3} .
\end{aligned}
$$

Then $\tilde{r}$ is given by $e \mapsto 12343578$ and $f \mapsto 1678$, and

$$
\begin{aligned}
& \tilde{s} \circ \tilde{r}: e \mapsto e_{1} e_{2} f_{1} f_{2} f_{3} f_{1} f_{2} f_{3} f_{1} f_{2} f_{3}=e f f f, \text { and } f \mapsto e_{1} e_{2} f_{1} f_{2} f_{3}=e f, \\
& \tilde{r} \circ \tilde{s}: 1 \mapsto 1234357,2,4,5,6 \mapsto 8,3 \mapsto 167,7 \mapsto 1, \text { and } 8 \mapsto 678 .
\end{aligned}
$$

Therefore we have $s \circ r=g_{\{p\}}$ and $r \circ s=g_{1}$, and $\left(Y_{\{p\}}, g_{\{p\}}\right)$ is shift equivalent to $\left(Y_{1}, g_{1}\right)$ by $r$ and $s$.

Now $\mathcal{P}_{\{x, y\}}=\{\alpha, \beta, \gamma\}$ where $\alpha$ is the path from $y$ through $p$ to $x, \beta$ is the circle $F$ based at $x$, and $\gamma$ is the path from $x$ through $p$ to $y$. And $\mathcal{P}_{\{u, v\}}=\{\delta, \epsilon, \zeta, \eta\}$ 
where $\delta$ is the path from $u$ through the path 2 to $v, \epsilon$ is the circle 34 based at $v$, $\zeta$ is the path from $v$ through the path 5 to $u$, and $\eta$ is the circle 1678 based at $u$. The wrapping rules $\tilde{g}_{\{x, y\}}: \mathcal{P}_{\{x, y\}} \rightarrow \mathcal{P}_{\{x, y\}}^{*}$ and $\tilde{g}_{\{u, v\}}: \mathcal{P}_{\{u, v\}} \rightarrow \mathcal{P}_{\{u, v\}}^{*}$ are given by

$$
\begin{aligned}
& \tilde{g}_{\{x, y\}}: \alpha \mapsto \beta \beta \gamma, \quad \beta \mapsto \alpha \gamma, \quad \gamma \mapsto \alpha \gamma \alpha, \text { and } \\
& \tilde{g}_{\{u, v\}}: \delta \mapsto \epsilon \zeta, \quad \epsilon \mapsto \eta, \quad \zeta \mapsto \eta \eta \delta, \quad \eta \mapsto \epsilon \zeta \eta \delta .
\end{aligned}
$$

Therefore the SFT covers associated to $\{x, y\}$ and $\{u, v\}$ are presented by the following matrices:

$$
M_{\{x, y\}}=\left(\begin{array}{ccc}
0 & 2 & 1 \\
1 & 0 & 1 \\
2 & 0 & 1
\end{array}\right), \quad M_{\{u, v\}}=\left(\begin{array}{cccc}
0 & 1 & 1 & 0 \\
0 & 0 & 0 & 1 \\
1 & 0 & 0 & 2 \\
1 & 1 & 1 & 1
\end{array}\right) .
$$

For

$$
R=\left(\begin{array}{llll}
0 & 1 & 1 & 0 \\
0 & 0 & 0 & 1 \\
1 & 0 & 0 & 1
\end{array}\right) \text { and } S=\left(\begin{array}{ccc}
1 & 0 & 0 \\
0 & 1 & 0 \\
0 & 1 & 1 \\
1 & 0 & 1
\end{array}\right)
$$

we have $M_{\{x, y\}}=R S$ and $M_{\{u, v\}}=S R$, and the two-sided SFT covers are topologically conjugate (as guaranteed by Theorem 5.2). On the other hand, Williams showed [17] that one-sided SFTs are conjugate if and only if they have the same total column amalgamation matrix; and we see that $M_{\{x, y\}}$ is its own total column amalgamation, whereas the total column amalgamation of $M_{\{u, v\}}$ is

$$
\left(\begin{array}{lll}
0 & 1 & 0 \\
1 & 0 & 3 \\
1 & 1 & 1
\end{array}\right)
$$

Therefore these one-sided SFTs are not conjugate.

\section{Appendix B. Relation Between Williams' Definition AND TOPOLOGICAL DEFINITION OF 1-SOLENOIDS}

Let $(X, f)$ be a presentation of a solenoid in our topological sense, that is, assume that it satisfies the Axioms 0-5 of Section 2. In this appendix, we will give $X$ the differentiable structure of a branched one-dimensional manifold, with respect to which $f$ will be an immersion. This will show that our topological 1-solenoids are exactly the systems obtained from Williams' differentiable 1-solenoids by forgetting the differentiable structure.

The precise definition of an $n$-dimensional branched manifold ([16]) is necessarily somewhat complicated and subtle. In our one-dimensional situation, we will attach to $(X, f)$ a more simple and special structure, from which an interested reader can easily derive the full immersion of a branched manifold apparatus laid out in [16]. There is some overlap in our ideas and those used by Williams for his Realization Theorem [15, 7.6].

We will cover the graph $X$ with open sets $W_{1}, \ldots, W_{k}$. Each $W_{i}$ will be an interval or a union of intervals intersecting at a vertex. There will be attached maps (charts) $\pi_{i}: W_{i} \rightarrow I_{i}$, where the $I_{i}$ are open intervals. When $W_{i}$ is an interval, $\pi_{i}$ will be a homeomorphism; in the other case $W_{i}$ will be a union of intervals the restriction of $\pi_{i}$ to each of which is a homeomorphism. Let $W_{i j}=W_{i} \cap W_{j}$. 
Whenever $i \neq j$ and $W_{i j}$ is nonempty, the restriction of the map $\pi_{i}$ to $W_{i j}$ will be invertible and the 'change-of-coordinates' map

$$
\pi_{i, j}: \pi_{i}\left(W_{i j}\right) \rightarrow \pi_{j}\left(W_{i j}\right) \text { given by } x \mapsto \pi_{j} \circ \pi_{i}^{-1} x
$$

will be invertible and affine. Finally, for each pair $i, j$ such that $f\left(W_{i}\right) \cap W_{j}$ is nonempty, there will be an invertible affine map $f_{i, j}: I_{i} \rightarrow I_{j}$ such that the restriction of $f$ to $W_{i} \cap f^{-1}\left(W_{j}\right)$ is a lift of $f_{i, j}$, that is to say, $f_{i, j} \circ \pi_{i}(x)=\pi_{j} \circ f(x)$ for all $x$ in $W_{i} \cap f^{-1}\left(W_{j}\right)$.

Recall that $\mathcal{V}$ is the vertex set of the graph $X$. To define the open sets $W_{i}$, fix $N$ such that each point of $X$ has a neighborhood whose image under $f^{N}$ is an interval such that the interior of every interval with endpoints in $f^{-1}(\mathcal{V})$ contains a point in $f^{-N}(\mathcal{V}) \backslash f^{-1}(\mathcal{V})$. The sets $W_{i}$ will be of two sorts. First, the complement of $f^{-N}(\mathcal{V})$ in $X$ will be a disjoint union of open intervals, and each of these will be one of the sets $W_{i}$. Second, at each point $y$ in $f^{-N}(\mathcal{V})$, we pick a connected open neighborhood $U_{y}$ such that $U_{y}$ are disjoint; after some shrinking, these will be the remaining $W_{i}$. The complement of $y$ in $U_{y}$ will be the union of a collection $\mathcal{J}(y)$ of disjoint open intervals $J_{y, t}$ (if $y$ is not a vertex, then there are just two); we pass to a shrunken set of $U_{y}$ for which the images of any pair $J_{y, t}$ and $J_{y^{\prime}, t^{\prime}}$ are equal or disjoint. This completes the description of the set $W_{i}$.

To describe the charts, we will use the following result of Williams. Williams proved it for elementary presentations, but his proof works for the non-elementary case and it works with our topological axioms.

Lemma B.1 ([15, 6.2]). There exist a unique measure $\mu$ on $X$ and a unique real number $\lambda>1$ such that $\mu(X)=1$ and $\mu(f(I))=\lambda I$ for every small interval contained in an edge.

First we use this measure $\mu$ to define the charts $\pi_{i}$ when $W_{i}$ is an interval: identifying $W_{i}$ with $(0,1)$, we define $\pi_{i}(x)=\mu(0, x)$. Next, for each $y$ in $f^{-N}(V)$, define an equivalence relation on $\mathcal{J}(y)$ by declaring two intervals to be equivalent if their images under $f^{N}$ are equal. This divides each $\mathcal{J}(y)$ into two equivalence classes; making an arbitrary choice, designate one class as $\mathcal{L}(y)$ and the other as $\mathcal{R}(y)$.

Now we can describe the chart $\pi_{i}$ for $W_{i}=U_{y}$. Identify each $J_{y, t}$ with the interval $(0,1)$, with 0 corresponding to $y$, and for $x$ in $J_{v, i}$ define

$$
\pi_{v}(x)= \begin{cases}\mu(0, x) & \text { if } J_{v, i} \in R(v), \\ -\mu(0, x) & \text { if } J_{v, i} \in L(v) .\end{cases}
$$

Finally, define $\pi_{i}(y)=0$. This completes the definition of the charts $\pi_{i}$. It is easy to verify that the change-of-coordinate maps $\pi_{i, j}$ are one-to-one and affine as claimed.

It remains to see that $f$ is locally the lift of affine maps as claimed. Suppose that the set $W_{i, j}=W_{i} \cap f^{-1}\left(W_{j}\right)$ is nonempty. If $W_{i}$ is not one of the $U_{y}$, then by choice of $N, f\left(W_{i}\right) \cap W_{j}$ is an interval, and by the choice of $N$ if $W_{j}=U_{y}$, then this interval is entirely contained in one of the intervals $J_{y, t}$; so the required affine map $f_{i, j}$ (with multiplicative constant $\lambda$ or $-\lambda$ ) exists. Similarly the required $f_{i, j}$ exists if $W_{i}=U_{y}$ and $f(y)$ is not a vertex.

Next, assume $W_{i}=U_{y}$ and $f(y)$ is a vertex. For each $J_{y, t}$, let $J_{t}^{\prime}$ denote the unique member of $\mathcal{J}(f(y))$ intersecting $f\left(J_{y, t}\right)$. First suppose that $y$ is not a vertex, so $\mathcal{J}(y)=\left\{J_{y, 1}, J_{y, 2}\right\}$. By the Nonfolding Axiom, the restriction of $f^{N+1}$ to $W_{i}$ 
is locally injective at $y$, and therefore $J_{1}^{\prime}$ and $J_{2}^{\prime}$ must be in different $\mathcal{L} / \mathcal{R}$ classes. Therefore the required affine map $f_{i, j}$ exists, with multiplicative constant $\lambda$ or $-\lambda$.

Finally, suppose $W_{i}=U_{y}, y$ is a vertex and (therefore) $f(y)$ is a vertex. We claim that for any $J_{1}$ in $\mathcal{L}(y)$ and $J_{2}$ in $\mathcal{R}(y)$, the intervals $J_{1}^{\prime}$ and $J_{2}^{\prime}$ cannot be in the same equivalence class $\mathcal{L}(f(y))$ or $\mathcal{R}(f(y))$. Given the claim, we can define the required map $f_{i, j}$ as $x \mapsto \lambda x$ (if the $\mathcal{L}, \mathcal{R}$ equivalence class labeling is respected) or $x \mapsto-\lambda x$ (if the labeling is reversed). So it remains to prove the claim.

Suppose the claim is false: suppose that there are $J_{1} \in \mathcal{L}(y)$ and $J_{2} \in \mathcal{R}(y)$ with $J_{1}^{\prime}$ and $J_{2}^{\prime}$ both in $\mathcal{L}(f(y))$ or both in $\mathcal{R}(f(y))$. Because $f^{N}\left(J_{1}^{\prime}\right)=f^{N}\left(J_{2}^{\prime}\right)$ it follows that $f^{N+1}$ is not locally injective at $y$ on the interval which is the union of $\{y\}$, $J_{1}$ and $J_{2}$. Since the image of an interval $J_{y, t}$ under $f^{N}$ depends only on the class $\mathcal{L}(y)$ or $\mathcal{R}(y)$ to which it belongs, it follows that the restriction of $f^{N+1}$ to any open interval containing $y$ is not locally one-to-one. Now pick a positive integer $k$ and a point $w$ which is not a vertex such that $f^{k}(w)=y$. Then $f^{k+N+1}$ is not locally injective at $w$. This contradicts the Nonfolding Axiom, and concludes the proof. We record the result as the following Proposition.

Proposition B.2. Suppose $(X, f)$ is a presentation of a topologically defined 1solenoid. Then $X$ can be given a differentiable structure making it a branched manifold on which $f$ is an immersion presenting a Williams solenoid.

Example B.3. Let $X$ be given by the following graph and $f: X \rightarrow X$ given by

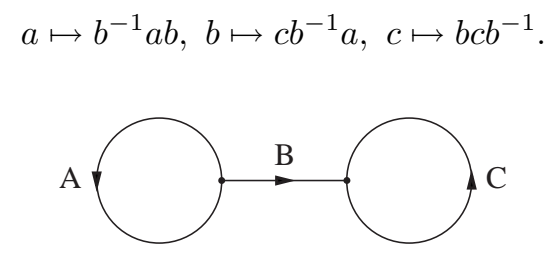

If we redraw $X$ as the following graph, then $X$ is a branched manifold, and $(X, f)$ is a presentation in the sense of Williams.

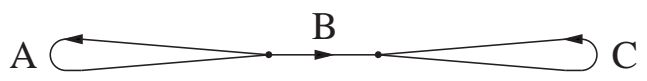

In contrast to the solenoid case, the following example shows that the set of Williams' branched solenoid systems is a proper subset of the topologically defined branched solenoid systems.

Example B.4. Let $X$ be as in the previous example, and $g: X \rightarrow X$ is given by

$$
a \mapsto c b^{-1} a b, b \mapsto c^{-1} b^{-1}, c \mapsto a .
$$

Then $(X, g)$ satisfies all Axioms except for the Flattening Axiom. So $(X, g)$ is a presentation of a branched solenoid according to the topological definition.

At each branch point of $X$, there are three choices of differentiable structure, that is, three arcs are parallel to each other or two arcs are parallel and the other arc is 180 degrees to these two arcs. The second graph in Example B.3 and graphs in Figure B.4 show three possible differentiable structures at the left branch point when the circle $c$ is fixed. And similar differentiable structures can be given to the right branch point. 

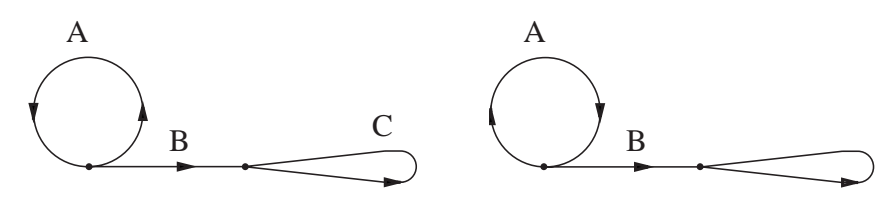

FiguRE 4. Other differentiable structures at the branch point of $A$ when the circle $C$ is fixed.

In each choice of differential structures at both branched points, it is not difficult to find a smooth interval which is mapped to a non-smooth interval homeomorphically by $g: X \rightarrow X$. For example, if $X$ is the second graph in Example B.3, then the interval $I$ is mapped to the interval $J$ by $g$ homeomorphically. Hence $(X, g)$ cannot be a presentation of a branched solenoid according to Williams' definition.

\section{ACKNOWLEDGMENT}

This paper is part of my Ph.D. thesis at UMCP. I would like to express deep gratitude to my advisor Dr. Mike Boyle for his encouragement and many useful discussions.

\section{REFERENCES}

1. R. Adler and B. Marcus, Topological entropy and equivalence of dynamical systems, Mem. Amer. Math. Soc. 219, 1979. MR 83h:28027

2. J. Anderson and I. Putnam, Topological invariants for substitution tilings and their associated $C^{*}$-algebras, Ergodic Th. and Dynam. Sys. 18 (1998), 509-37. MR 2000a:46112

3. M. Barge, J. Jacklitch, and G. Vago, Homeomorphisms of One-dimensional Inverse Limits with Applications to Substitution Tilings, Unstable Manifolds, and Tent Maps, Geometry and Topology in Dynamics, 1-15, Contemp. Math. 246, Amer. Math. Soc., 1999. MR 2000j:37016

4. R. Bowen, Equilibrium states and the ergodic theory of Anosov diffeomorphisms, Lecture Notes in Math. 470, Springer-Verlag, 1975. MR 56:1364]

5. R. Bowen and J. Franks, Homology for zero-dimensional nonwandering sets, Ann. of Math. 106, (1977), 73-92. MR 56:16692

6. F. Durand, B. Host and C. Skau, Substitution dynamical systems, Bratteli diagrams and dimension groups, Ergodic Th. and Dynam. Sys. 19 (1999), 953-993. MR 2000i:46062

7. D. Fried, Finitely presented dynamical systems, Ergodic Th. and Dynamical Sys. 7 (1987), 489-507. MR 89h:58157

8. W. Krieger, On sofic systems I, Israel J. Math. 48 (1984), 305-330. MR 86j:54074

9. D. Lind and B. Marcus, An introduction to symbolic dynamics and coding, Cambridge University Press, 1995. MR 97a:58050

10. C. Robinson, Dynamical systems: stability, symbolic dynamics, and chaos (2nd Edition), CRC Press, 1999.

11. M. Snavely, Markov partitions for the two-dimensional torus, Proc. Amer. Math. Soc. 113 (1991), 517-527. MR 91m:58122

12. R. Swanson and H. Volkmer, Invariants of weak equivalence in primitive matrices, Ergodic Th. and Dynam. Sys. 20 (2000), 611-626. CMP 2000:12

13. Y. Ustinov, Algebraic invariants of topological conjugacy of solenoids, Mat. Zametki 42 (1987), 132-144. MR 89g:58167

14. R. F. Williams, One-dimensional non-wandering sets, Topology 6 (1967), 473-487.

15. R. F. Williams, Classification of 1-dimensional attractors, Proc. Symp. Pure Math. 14 (1970), 341-361.

16. R. F. Williams, Expanding attractors, IHES Publ. Math. 43 (1974), 169-203. MR 36:897 
17. R. F. Williams, Classification of subshifts of finite type, Ann. of Math. (2) 98 (1973), 120-153; errata, ibid. (2) 99 (1974), 380-381. MR 48:9769

18. I. Yi, Ordered group invariants for one-dimensional spaces, Preprint, 2000

19. I. Yi, Orientable double cover and Bratteli-Vershik systems for one-dimensional generalized solenoids, Preprint, 2000.

Department of Mathematics, University of Maryland, College Park, Maryland 20742

E-mail address: inhyeop@math.umd.edu 